\title{
QUANTUM E(2) GROUPS FOR COMPLEX DEFORMATION PARAMETERS
}

\author{
ATIBUR RAHAMAN AND SUTANU ROY
}

\begin{abstract}
We construct a family of $q$ deformations of $\mathrm{E}(2)$ group for nonzero complex parameters $|q|<1$ as locally compact braided quantum groups over the circle group $\mathbb{T}$ viewed as a quasitriangular quantum group with respect to the unitary $R$-matrix $\mathrm{R}(m, n):=(q / \bar{q})^{m n}$ for all $m, n \in \mathbb{Z}$. For real $0<|q|<1$, the deformation coincides with Woronowicz's $\mathrm{E}_{\mathrm{q}}(2)$ groups. As an application, we study the braided analogue of the contraction procedure between $\mathrm{SU}_{q}(2)$ and $\mathrm{E}_{q}(2)$ groups in the spirit of Woronowicz's quantum analogue of the classic Inönü-Wigner group contraction. Consequently, we obtain the bosonisation of braided $\mathrm{E}_{q}(2)$ groups by contracting $\mathrm{U}_{q}(2)$ groups.
\end{abstract}

\section{INTRODUCTION}

Quantum E(2) groups were constructed as $q$-deformations of the double cover of the group of motions of the Euclidean plane $\mathrm{E}(2)$ for real deformation parameters $0<q<1[22,23]$. They were used as a prototype for constructing a large class of examples of locally compact quantum groups [28,29. Here we construct a family of $q$-deformations of the same group for complex deformation parameters $0<|q|<1$. Consider the pair of operators $(v, n)$ satisfying the following conditions

$$
v \text { is unitary, } \quad n \text { is normal, } \quad v n v^{*}=q n, \quad \operatorname{Sp}(n)=\overline{\mathbb{C}}^{|q|},
$$

where $\overline{\mathbb{C}}^{|q|}:=\left\{\lambda \in \mathbb{C}:|\lambda| \in|q|^{\mathbb{Z}}\right\} \cup\{0\}$. Let $q=|q| \Phi_{q}$ and $n=|n| \Phi_{n}$ be the polar decompositions of $q$ and $n$, respectively. The explicit meaning of the commutation relation $v n v^{*}=q n$ is $v \Phi_{n} v^{*}=\Phi_{q} \Phi_{n}$ and $v|n| v^{*}=|q||n|$.

The spectral condition of $n$ implies that $\mathrm{C}_{0}\left(\overline{\mathbb{C}}^{|q|}\right)$ is generated by the unbounded normal operator $n$ in the sense of [26, Definition 3.1]. The commutation relation $v n v^{*}=q n$ induces the following action $\alpha: \mathbb{Z} \rightarrow \operatorname{Aut}\left(\mathrm{C}_{0}\left(\overline{\mathbb{C}}^{|q|}\right)\right)$ :

$$
\alpha_{k}(f)(\lambda)=f\left(q^{k} \lambda\right), \quad \text { for all } k \in \mathbb{Z}, f \in \mathrm{C}_{0}\left(\overline{\mathbb{C}}^{|q|}\right) \text { and } \lambda \in \overline{\mathbb{C}}^{|q|} .
$$

Let $B=\mathrm{C}_{0}\left(\overline{\mathbb{C}}^{|q|}\right) \rtimes_{\alpha} \mathbb{Z}$ be the associated crossed product $\mathrm{C}^{*}$-algebra. By virtue of [23. Theorem 1.6] and the universal property of the $\mathrm{C}^{*}$-algebra crossed products, the pair of operators $(v, n)$ in (1.1) generate the $\mathrm{C}^{*}$-algebra $B$. In particular, $v, n \notin B$ but these are affiliated with $B$, see [23, Definition 1.1].

For real $0<q<1$, one has a well-defined nondegenerate ${ }^{*}$-homomorphism $\Delta_{B}: B \rightarrow \mathcal{M}(B \otimes B)$ satisfying

$$
\Delta_{B}(v)=v \otimes v, \quad \Delta_{B}(n)=v \otimes n \dot{+} n \otimes v^{*} .
$$

2020 Mathematics Subject Classification. Primary: 46L89; secondary: 81R50, 18M15.

Key words and phrases. braided multiplicative unitary, braided quantum E(2) group, quasitriangular quantum group, R-matrix, bosonisation, contraction.

The first author was supported by a UGC NET-JRF fellowship, India. The second author was partially supported by an Early Career Research Award given by SERB-DST, Government of India Grant No. ECR/2017/001354. 
Here $\otimes, \mathcal{M}(B)$ and $\dot{+}$ denote the minimal tensor product of $\mathrm{C}^{*}$-algebras, multiplier algebra of $B$ and the closure of the sum of the operators, respectively. Also, $\Delta_{B}(n)$ is affiliated with $B \otimes B$. The pair $\left(B, \Delta_{B}\right)$ is Woronowicz's $\mathrm{E}_{\mathrm{q}}(2)$ group [22,23].

However, the passage from the real to the complex deformation parameter $q$ reveals two interesting features. Firstly, the $\mathrm{C}^{*}$-algebra $B$ for the deformation parameter $|q|$ is isomorphic to the one for the complex parameter $q$, see Proposition 5.1. Secondly, $\Delta_{B}(n)$ and $\Delta_{B}(n)^{*}$ do not commute (even formally). In other words, $\left(B, \Delta_{B}\right)$ fails to be a $C^{*}$-quantum group unless $q=\bar{q}$.

We address this issue by replacing the ordinary tensor product $\otimes$ by the twisted tensor product $\otimes_{\mathrm{R}}$ from [14] associated to the bicharacter $\mathrm{R}: \mathbb{Z} \times \mathbb{Z} \rightarrow \mathbb{T}$ defined by

$$
\mathrm{R}(m, n):=\zeta^{m n}, \quad \text { for all } m, n \in \mathbb{Z} \text { with } \zeta:=\frac{q}{\bar{q}}=\left(\Phi_{q}\right)^{2} .
$$

For that matter, we view the circle group $\mathbb{T}$ as a quasitriangular quantum group with respect to $\mathrm{R}$ as explained in Example 2.11.

Now consider the category $\mathfrak{C}^{*} \mathfrak{a l g}(\mathbb{T})$ whose objects are $\mathrm{C}^{*}$-algebras equipped with an action of $\mathbb{T}$ and morphisms are $\mathbb{T}$-equivariant nondegenerate ${ }^{*}$-homomorphisms. Clearly, $\mathrm{C}(\mathbb{T})$ is an object of $\mathfrak{C}^{*} \mathfrak{a l g}(\mathbb{T})$ with respect to the translation action of $\mathbb{T}$. Also, the universal property of $B$ ensures that $(v, \lambda) \rightarrow v$ and $(n, \lambda) \rightarrow \lambda n$ for all $\lambda \in \mathbb{T}$ extends uniquely to an action of $\mathbb{T}$ on $B$ (see also (5.5)). The monoidal structure $\bigotimes_{\mathrm{R}}$ on $\mathfrak{C}^{*} \mathfrak{a l g}(\mathbb{T})$ is defined as follows: $C \bigotimes_{\mathrm{R}} D$ is a $C^{*}$-algebra for all objects $\left(C, \delta_{C}\right),\left(D, \delta_{D}\right)$ of $\mathfrak{C}^{*} \mathfrak{a l g}(\mathbb{T})$ with injective nondegenerate *-homomorphisms $j_{1}: C \rightarrow \mathcal{M}\left(C \otimes_{\mathrm{R}} D\right)$ and $j_{2}: D \rightarrow \mathcal{M}\left(C \otimes_{\mathrm{R}} D\right)$ such that the *-algebra generated by $\left\{j_{1}(c) j_{2}(d) \mid c \in C, d \in D\right\}$ is dense in $C \bigotimes_{\mathrm{R}} D$. On the homogeneous elements $c_{k} \in C$ and $d_{l} \in D$ of degree $k$ and $l$, that is $\delta_{C}\left(c_{k}\right)=c_{k} \otimes$ $z^{k}$ and $\delta_{D}\left(d_{l}\right)=d_{l} \otimes z^{l}$, the canonical embeddings $j_{1}, j_{2}$ commute up to $\zeta^{k l}$ : $j_{1}\left(c_{k}\right) j_{2}\left(d_{l}\right)=\zeta^{k l} j_{2}\left(d_{l}\right) j_{1}\left(c_{k}\right)$.

In particular, we consider the twofold twisted tensor product $B \nabla_{\mathrm{R}} B$ and define an analogue of $(1.3)$ in $\mathfrak{C}^{*} \mathfrak{a l g}(\mathbb{T})$ by

$$
\Delta_{B}(v):=j_{1}(v) j_{2}(v), \quad \Delta_{B}(n):=j_{1}(v) j_{2}(n) \dot{+} j_{1}(n) j_{2}\left(v^{*}\right) .
$$

The primary goal of this article is to prove that $\mathrm{E}_{\mathrm{q}}(2)=\left(B, \Delta_{B}\right)$ is a braided $\mathrm{C}^{*}$-quantum group over the quasitriangular quantum group $\mathbb{T}$ with respect to $\mathrm{R}$. This is essentially contained in Theorem 5.9. Subsequently, we construct an ordinary $\mathrm{C}^{*}$-quantum group $\mathbb{H}=\left(C, \Delta_{C}\right)$ with an idempotent quantum group homomorphism with image $\mathbb{T}$. In the Hopf-algebraic context this process is known as bosonisation which was discovered by Radford [17] and extensively studied by Majid 911 . Therefore, $\mathbb{H}=\left(C, \Delta_{C}\right)$ is the analytic counter part of the bosonisation of $\mathrm{E}_{\mathrm{q}}(2)$, which is a new example of (non-compact) $\mathrm{C}^{*}$-quantum group.

The double cover of $\mathrm{E}(2)$ may be constructed by contracting $\mathrm{SU}(2)$. This is one of the well studied examples of the classic Inönü-Wigner group contraction [7]. In the purely algebraic setting, the quantum analogue of the contraction procedure was investigated by Celeghini, Giachetti, Sorace and Tarlini 3 -5 and applied to construct new examples of quantum groups. Their work motivated Woronowicz [25] to study the contraction procedure for $\mathrm{C}^{*}$-quantum groups. In this article, it was shown that for real $0<q<1$, the contraction of the compact quantum groups $\mathrm{SU}_{\mathrm{q}}(2)$ respect to the (closed quantum) subgroup $\mathbb{T}$ coincides with $\mathrm{E}_{\mathrm{q}}(2)$ groups.

Deformations of SU(2) group for complex $q$ satisfying $0<|q|<1$ was considered in [8]. They are braided compact quantum groups over the quasitriangular quantum group $\mathbb{T}$ with respect to $\mathrm{R}$ and $\mathrm{U}_{q}(2)$ groups are the associated bosonisation. Now $\mathbb{T}$ is a closed quantum subgroup of braided $\mathrm{SU}_{\mathrm{q}}(2)$ groups [20, Proposition 4.1] in the sense of Woronowicz [6, Definition 3.2]. Similarly, we observe that $\mathbb{T}$ is also a closed quantum subgroup of braided $\mathrm{E}_{\mathrm{q}}(2)$ in Remark 5.10 , Then we prove a braided 
analogue of the contraction procedure between $\mathrm{SU}_{\mathrm{q}}(2)$ and $\mathrm{E}_{\mathrm{q}}(2)$ in Theorem 6.6 More precisely, the $\mathbb{T}$-equivariant contraction of braided $\mathrm{SU}_{\mathrm{q}}(2)$ groups with respect to $\mathbb{T}$ are isomorphic to braided $\mathrm{E}_{\mathrm{q}}(2)$ groups. Consequently, we show that $\mathbb{H}$ can be constructed by contracting $\mathrm{U}_{q}(2)$ with respect to the same subgroup $\mathbb{T}$.

Let us briefly outline the structure of this article. We have gathered all the necessary preliminaries in Section 2, In general, the category of Yetter-Drinfeld represenations of a $\mathrm{C}^{*}$-quantum group $\mathbb{G}$, denoted by $\mathcal{Y} \mathcal{D} \mathfrak{R e}(\mathbb{G})$, is a braided monoidal category, and additionally the braiding isomorphisms are unitary operators. In short, we call such a category as a unitarily braided monoidal category. Motivated by [27, a general theory of braided $\mathrm{C}^{*}$-quantum groups [19] is developed using manageable braided multiplicative unitaries [15,18, in $\mathcal{Y} \mathcal{D} \mathfrak{R e p}(\mathbb{G})$, where $\mathbb{G}$ is a regular $\mathrm{C}^{*}$-quantum group.

Suppose $\mathbb{G}=\left(A, \Delta_{A}\right)$ is a regular quasitriangular $\mathrm{C}^{*}$-quantum group with respect to a unitary $R$-matrix $\mathrm{R} \in \mathcal{U}(\hat{A} \otimes \hat{A})$, where $\widehat{\mathbb{G}}=\left(\hat{A}, \hat{\Delta}_{A}\right)$ is the dual of $\mathbb{G}$. Then the representation category $\mathfrak{R e p}(\mathbb{G})$ of $\mathbb{G}$ is a unitarily braided monoidal category [14, Section 3]. Therefore, it is natural to work with manageable braided multiplicative unitaries in $\mathfrak{R e p}(\mathbb{G})$ instead of $\mathcal{Y} \mathcal{D} \mathfrak{R} \mathfrak{e}(\mathbb{G})$ whenever $\mathbb{G}$ is a quasitriangular quantum group and construct braided $\mathrm{C}^{*}$-quantum groups out of them. This is done in Section 3 using the results presented in the Appendix $\mathrm{A}$ dealing with the Yetter-Drinfeld representations of $\mathbb{G}$. In particular, the construction of braided $\mathrm{C}^{*}$-quantum groups over $\mathbb{G}$ is presented in Theorem 3.6.

From Section 4 onwards we fix $\mathbb{G}$ to be the circle group $\mathbb{T}$ (viewed as a quantum group) and the $R$-matrix $\mathrm{R}$ defined in (1.4). Let $\mathcal{L}$ be a Hilbert space equipped

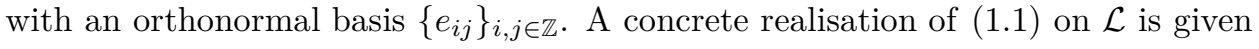
by

$$
v e_{i, j}:=e_{i-1, j}, \quad n e_{i, j}:=q^{i} e_{i, j+1} .
$$

In fact, any Hilbert space realisation of (1.1) is either one dimensional or infinite dimensional, and the direct integral of all infinite dimensional irreducible representations of (1.1) is unitarily equivalent to (1.6), see [23, Section 3 (B)]. Therefore, (1.6) defines a faithful nondegenerate *-representation of $B$ on $\mathcal{L}$.

We identify $\mathcal{L} \cong \ell^{2}(\mathbb{Z}) \otimes \ell^{2}(\mathbb{Z})$ and use the canonical representation of $\mathbb{T}$ on the second tensor factor. This makes $\mathcal{L}$ an object of $\mathfrak{R e p}(\mathbb{T})$ and $\mathrm{R}$ induces the unitary braiding $\chi \in \mathcal{U}(\mathcal{L} \otimes \mathcal{L})$. On standard basis elements $\left\{e_{i, j} \otimes e_{k, l}\right\}_{i, j, k, l \in \mathbb{Z}}$ of $\mathcal{L} \otimes \mathcal{L}$ the action of $\chi$ is given by $e_{i, j} \otimes e_{k, l} \rightarrow \zeta^{-j l} e_{k, l} \otimes e_{i, j}$. Starting with these data, we construct a manageable braided multiplicative unitary $\mathbb{F} \in \mathcal{U}(\mathcal{L} \otimes \mathcal{L})$ in the category $\mathfrak{R e p}(\mathbb{T})$, see Theorem 4.6 .

The main results of this article are presented in Section 5 . We apply Theorem 3.6 to $\mathbb{F}$ and construct $\mathrm{E}_{\mathrm{q}}(2)=\left(B, \Delta_{B}\right)$ as a braided group over $\mathbb{T}$ from it in Theorem 5.9. The associated bosonisation $\mathbb{H}=\left(C, \Delta_{C}\right)$ is described in Theorem 5.15] In the final section $\left[\right.$, we present the contraction procedure between $\mathbb{H}$ and $\mathrm{U}_{q}(2)$ group.

\section{Preliminaries}

All Hilbert spaces and $\mathrm{C}^{*}$-algebras (which are not explicitly multiplier algebras) are assumed to be separable. For a $\mathrm{C}^{*}$-algebra $A$, let $\mathcal{M}(A)$ be its multiplier algebra and let $\mathcal{U}(A)$ be the group of unitary multipliers of $A$. The unit of $\mathcal{M}(A)$ is denoted by $1_{A}$. For two norm closed subsets $X$ and $Y$ of a $\mathrm{C}^{*}$-algebra $A$ and $T \in \mathcal{M}(A)$, set

$$
X T Y:=\{x T y: x \in X, y \in Y\}^{- \text {closed linear span }} .
$$

Let $\mathfrak{C}^{*} \mathfrak{a l g}$ be the category of $\mathrm{C}^{*}$-algebras with nondegenerate *-homomorphisms $\varphi: A \rightarrow \mathcal{M}(B)$ as morphisms $A \rightarrow B$; let $\operatorname{Mor}(A, B)$ denote the set of morphisms. 
We use the same symbol for an element of $\operatorname{Mor}(A, B)$ and its unique unital extension from $\mathcal{M}(A)$ to $\mathcal{M}(B)$.

Let $\mathcal{H}$ be a Hilbert space. A representation of a $\mathrm{C}^{*}$-algebra $A$ is a nondegenerate ${ }^{*}$-homomorphism $\pi: A \rightarrow \mathbb{B}(\mathcal{H})$. Since $\mathbb{B}(\mathcal{H})=\mathcal{M}(\mathbb{K}(\mathcal{H}))$ and the nondegeneracy conditions $\pi(A) \mathbb{K}(\mathcal{H})=\mathbb{K}(\mathcal{H})$ and $\pi(A) \mathcal{H}=\mathcal{H}$ are equivalent; hence $\pi \in \operatorname{Mor}(A, \mathbb{K}(\mathcal{H}))$. The identity operator on $\mathcal{H}$ is denoted by id $\mathcal{H}$. Whereas the the unit element of $\mathcal{M}(\mathbb{K}(\mathcal{H}))=\mathbb{B}(\mathcal{H})$ is denoted by $1_{\mathcal{H}}$.

We write $\Sigma$ for the tensor flip $\mathcal{H} \otimes \mathcal{K} \rightarrow \mathcal{K} \otimes \mathcal{H}, x \otimes y \mapsto y \otimes x$, for two Hilbert spaces $\mathcal{H}$ and $\mathcal{K}$. We write $\sigma$ for the tensor flip isomorphism $A \otimes B \rightarrow B \otimes A$ for two $\mathrm{C}^{*}$-algebras $A$ and $B$.

2.1. $\mathbf{C}^{*}$-quantum groups, actions and representations. Let $\mathcal{H}$ be a Hilbert space. An element $\mathbb{W} \in \mathcal{U}(\mathcal{H} \otimes \mathcal{H})$ is called a multiplicative unitary [2, Definition $1.1]$ if it satisfies the pentagon equation

$$
\mathbb{W}_{23} \mathbb{W}_{12}=\mathbb{W}_{12} \mathbb{W}_{13} \mathbb{W}_{23} \quad \text { in } \mathcal{U}(\mathcal{H} \otimes \mathcal{H} \otimes \mathcal{H})
$$

Furthermore, $\mathbb{W} \in \mathcal{U}(\mathcal{H} \otimes \mathcal{H})$ is said to be manageable if there is a strictly positive operator $Q$ on $\mathcal{H}$ and a unitary $\widetilde{\mathbb{W}} \in \mathcal{U}(\overline{\mathcal{H}} \otimes \mathcal{H})$ satisfying

$$
\mathbb{W}(Q \otimes Q) \mathbb{W}^{*}=Q \otimes Q, \quad\langle x \otimes u|\mathbb{W}| z \otimes y\rangle=\left\langle\bar{z} \otimes Q u|\widetilde{\mathbb{W}}| \bar{x} \otimes Q^{-1} y\right\rangle
$$

for all $x, z \in \mathcal{H}, u \in \mathcal{D}(Q)$ and $y \in \mathcal{D}\left(Q^{-1}\right.$ ) (see [27, Definition 1.2]). Here $\overline{\mathcal{H}}$ is the conjugate Hilbert space, and an operator is strictly positive if it is positive and self-adjoint with trivial kernel. The condition $\mathbb{W}(Q \otimes Q) \mathbb{W}^{*}=Q \otimes Q$ means that the unitary $\mathbb{W}$ commutes with the unbounded operator $Q \otimes Q$.

A $\mathrm{C}^{*}$-quantum group $\mathbb{G}=\left(A, \Delta_{A}\right)$ consists of a $\mathrm{C}^{*}$-algebra $A$ and an element $\Delta_{A} \in \operatorname{Mor}(A, A \otimes A)$ constructed from a manageable multiplicative unitary $\mathbb{W}\left[27\right.$, Theorem 1.5]. Let $\widehat{\mathbb{G}}=\left(\hat{A}, \hat{\Delta}_{A}\right)$ be the dual $\mathrm{C}^{*}$-quantum group and let $\mathrm{W} \in \mathcal{U}(\hat{A} \otimes A)$ be the reduced bicharacter. In particular,

$$
\begin{aligned}
& A:=\left\{\left(\omega \otimes \mathrm{id}_{A}\right) \mathrm{W} \mid \omega \in \hat{A}^{\prime}\right\}^{- \text {closed linear span }} \subset \mathbb{B}(\mathcal{H}), \\
& \hat{A}:=\left\{\left(\mathrm{id}_{\hat{A}} \otimes \omega\right) \mathrm{W} \mid \omega \in A^{\prime}\right\}^{\text {-closed linear span }} \subset \mathbb{B}(\mathcal{H}) .
\end{aligned}
$$

Moreover, $\Delta_{A}$ and $\hat{\Delta}_{A}$ are characterised by

$$
\begin{array}{ll}
\left(\mathrm{id}_{\hat{A}} \otimes \Delta_{A}\right) \mathrm{W}=\mathrm{W}_{12} \mathrm{~W}_{13} & \text { in } \mathcal{U}(\hat{A} \otimes A \otimes A), \\
\left(\hat{\Delta}_{A} \otimes \mathrm{id}_{A}\right) \mathrm{W}=\mathrm{W}_{23} \mathrm{~W}_{13} & \text { in } \mathcal{U}(\hat{A} \otimes \hat{A} \otimes A) .
\end{array}
$$

We reserve the phrase "quantum groups" for $\mathrm{C}^{*}$-quantum groups. An important concept in the theory of quantum groups is the concept of regularity introduced by Baaj and Skandalis in [2]. A quantum group $\mathbb{G}=\left(A, \Delta_{A}\right)$ is said to be regular if $\left(\hat{A} \otimes 1_{A}\right) \mathrm{W}\left(1_{\hat{A}} \otimes A\right)=\hat{A} \otimes A$. Dual of a regular quantum group is again regular. A (right) action of $\mathbb{G}=\left(A, \Delta_{A}\right)$ on a $\mathrm{C}^{*}$-algebra $C$ is an injective morphism $\delta: C \rightarrow C \otimes A$ with the following properties:

(1) $\delta$ is a comodule structure, that is,

$$
\left(\operatorname{id}_{C} \otimes \Delta_{A}\right) \circ \delta=\left(\delta \otimes \operatorname{id}_{A}\right) \circ \delta ;
$$

(2) $\delta$ satisfies the Podleś condition:

$$
\delta(C)\left(1_{C} \otimes A\right)=C \otimes A .
$$

We call $(C, \delta)$ a $\mathbb{G}-\mathrm{C}^{*}$-algebra. We shall drop $\delta$ from our notation whenever it is clear from the context. A morphism $f: C \rightarrow D$ between two $\mathbb{G}$ - $\mathrm{C}^{*}$-algebras $\left(C, \delta_{C}\right)$ and $\left(D, \delta_{D}\right)$ is $\mathbb{G}$-equivariant if $\delta_{D} \circ f=\left(f \otimes \operatorname{id}_{A}\right) \circ \delta_{C}$. Let $\operatorname{Mor}^{\mathbb{G}}(C, D)$ be the set of $\mathbb{G}$-equivariant morphisms from $C$ to $D$. Let $\mathfrak{C}^{*} \mathfrak{a l g}(\mathbb{G})$ be the category with $\mathbb{G}-\mathrm{C}^{*}$-algebras as objects and $\mathbb{G}$-equivariant morphisms as arrows. 
A (right) representation of $\mathbb{G}$ on a $\mathrm{C}^{*}$-algebra $D$ is an element $\mathrm{U} \in \mathcal{U}(D \otimes A)$ with

$$
\left(\operatorname{id}_{D} \otimes \Delta_{A}\right) \mathrm{U}=\mathrm{U}_{12} \mathrm{U}_{13} \quad \text { in } \mathcal{U}(D \otimes A \otimes A) .
$$

In particular, if $D=\mathbb{K}(\mathcal{L})$ for some Hilbert space $\mathcal{L}$, then $\mathrm{U}$ is said to be a (right) representation of $\mathbb{G}$ on $\mathcal{L}$.

The tensor product of representations $\mathrm{U}^{i} \in \mathcal{U}\left(\mathbb{K}\left(\mathcal{L}_{i}\right) \otimes A\right)$ for $i=1,2$ is defined by $\mathrm{U}_{13}^{1} \mathrm{U}_{23}^{2} \in \mathcal{U}\left(\mathbb{K}\left(\mathcal{L}_{1} \otimes \mathcal{L}_{2}\right) \otimes A\right)$. It is denoted by $\mathrm{U}^{1} \oplus \mathrm{U}^{2}$. An element $t \in \mathbb{B}\left(\mathcal{L}_{1}, \mathcal{L}_{2}\right)$ is called an intertwiner if $\left(t \otimes 1_{A}\right) \mathrm{U}^{1}=\mathrm{U}^{2}\left(t \otimes 1_{A}\right)$. The set of all intertwiners between $\mathrm{U}^{1}$ and $\mathrm{U}^{2}$ is denoted by $\operatorname{Hom}^{\mathbb{G}}\left(\mathrm{U}^{1}, \mathrm{U}^{2}\right)$. A routine computation shows that $\oplus$ is associative; and the trivial 1-dimensional representation is a tensor unit. This gives representations a structure of $\mathrm{W}^{*}$-category, which we denote by $\mathfrak{R e} \mathfrak{p}(\mathbb{G})$; see [21, Section 3.1-2] for more details. Objects of $\mathfrak{R e p}(\mathbb{G})$ are pairs $(\mathcal{L}, \mathrm{U})$ consisting of a Hilbert space $\mathcal{L}$ and a representation $\mathrm{U}$ of $\mathbb{G}$ on $\mathcal{L}$.

A covariant representation of $(C, \delta, \mathbb{G})$ on a Hilbert space $\mathcal{L}$ is a pair $(\mathrm{U}, \varphi)$ consisting of a representation $\mathrm{U} \in \mathcal{U}(\mathbb{K}(\mathcal{L}) \otimes A)$ of $\mathbb{G}$ and a representation $\varphi: C \rightarrow$ $\mathbb{B}(\mathcal{L})$ of $C$ that satisfy the covariance condition

$$
\left(\varphi \otimes \operatorname{id}_{A}\right) \delta(c)=\mathrm{U}\left(\varphi(c) \otimes 1_{A}\right) \mathrm{U}^{*} \quad \text { in } \mathcal{U}(\mathbb{K}(\mathcal{L}) \otimes A)
$$

for all $c \in C$. A covariant representation is called faithful if $\varphi$ is faithful. Faithful covariant representations always exists, see for instance [13, Example 4.4].

Example 2.9. Let $G$ be a locally compact group. Let $\mathcal{H}:=L^{2}(G)$ with respect to the right Haar measure on $G$. A routine computation shows that the operator $(\mathbb{W} \xi)\left(g_{1}, g_{2}\right):=\xi\left(g_{1} g_{2}, g_{2}\right)$ for all $\xi \in L^{2}(G \times G)$ is a manageable multiplicative unitary with $Q=\operatorname{id}_{\mathcal{H}}$ and $\widetilde{\mathbb{W}}=\mathbb{W}^{*}$ and generates the quantum group $\mathbb{G}=$ $\left(\mathrm{C}_{0}(G), \Delta_{\mathrm{C}_{0}(G)}\right)$ where $\left(\Delta_{\mathrm{C}_{0}(G)} f\right)\left(g_{1}, g_{2}\right):=f\left(g_{1} g_{2}\right)$ for all $f \in \mathrm{C}_{0}(G)$. In this way, $G$ is viewed as a quantum group. Moreover, the category $\mathfrak{C}^{*} \mathfrak{a l g}(\mathbb{G})$ is equivalent to the category $\mathfrak{C}^{*} \mathfrak{a l g}(G)$ with $G$-C $\mathrm{C}^{*}$-algebras as objects and $G$-equivariant morphisms as arrows. Similarly, $\mathfrak{R e p}(\mathbb{G})$ is also equivalent to the representation category $\mathfrak{R e p}(G)$ of $G$. Let $\mu$ be the right regular representation of $G$ on $\mathcal{H}$. The dual of $\mathbb{G}$ is $\widehat{\mathbb{G}}=\left(\mathrm{C}_{\mathrm{r}}^{*}(G), \Delta_{\mathrm{C}_{\mathrm{r}}^{*}(G)}\right)$, where $\Delta_{\mathrm{C}_{\mathrm{r}}^{*}(G)}\left(\mu_{g}\right):=\mu_{g} \otimes \mu_{g}$. Also, $\mathbb{G}$ and $\widehat{\mathbb{G}}$ are regular quantum groups, and in addition, $\widehat{\mathbb{G}}$ coincides with $\hat{G}$ as quantum group whenever $G$ is Abelian.

For simplicity, whenever a locally compact group $G$ is viewed as a quantum group, we use the same notation $G$ to denote corresponding quantum group $\mathbb{G}=$ $\left(\mathrm{C}_{0}(G), \Delta_{\mathrm{C}_{0}(G)}\right)$.

2.2. Quasitriangular quantum groups. Let $\mathbb{G}=\left(A, \Delta_{A}\right)$ be a quantum group and let $\widehat{\mathbb{G}}=\left(\hat{A}, \hat{\Delta}_{A}\right)$ be the dual. An element $\mathrm{R} \in \mathcal{U}(\hat{A} \otimes \hat{A})$ is said to be an $R$-matrix on $\widehat{\mathbb{G}}$ if it is a bicharacter:

$$
\left(\operatorname{id}_{\hat{A}} \otimes \hat{\Delta}_{A}\right) \mathrm{R}=\mathrm{R}_{12} \mathrm{R}_{13}, \quad\left(\hat{\Delta}_{A} \otimes \operatorname{id}_{\hat{A}}\right) \mathrm{R}=\mathrm{R}_{23} \mathrm{R}_{13}, \quad \text { in } \mathcal{U}(\hat{A} \otimes \hat{A} \otimes \hat{A}) ;
$$

and satisfies the $R$-matrix condition:

$$
\mathrm{R}\left(\sigma \hat{\Delta}_{A}(\hat{a})\right) \mathrm{R}^{*}=\hat{\Delta}_{A}(\hat{a}) \quad \text { for all } \hat{a} \in \hat{A} .
$$

A quasitriangular quantum group is a quantum group $\mathbb{G}=\left(A, \Delta_{A}\right)$ with an $R$-matrix $\mathrm{R} \in \mathcal{U}(\hat{A} \otimes \hat{A})$, see [14, Definition 3.1].

Example 2.11. Every continuous bicharacter $\mathbb{Z} \times \mathbb{Z} \rightarrow \mathbb{T}$ satisfies the $R$-matrix condition (2.10) because $\hat{A}=\mathrm{C}_{0}(\mathbb{Z})$ is a commutative $\mathrm{C}^{*}$-algebra. Thus $\mathbb{T}$ is quasitringular with respect to any bicharacter on $\mathbb{Z} \times \mathbb{Z}$. In particular, for $q \in \mathbb{C} \backslash\{0\}$ the bicharacter $\mathrm{R}: \mathbb{Z} \times \mathbb{Z} \rightarrow \mathbb{T}$ in (1.4) is an $R$-matrix on $\mathbb{Z}$. 
Throughout this subsection we assume $\mathbb{G}=\left(A, \Delta_{A}\right)$ is a quasitriangular quantum group with an $R$-matrix $\mathrm{R} \in \mathcal{U}(\hat{A} \otimes \hat{A})$. Then the categories $\mathfrak{R e p}(\mathbb{G})$ and $\mathfrak{C}^{*} \mathfrak{a l g}(\mathbb{G})$ are of particular interest. More precisely, [14, Proposition $3.2 \&$ Theorem 4.3] show that $\mathfrak{R e p}(\mathbb{G})$ is a unitarily braided monoidal category and $\mathfrak{C}^{*} \mathfrak{a l g}(\mathbb{G})$ is a monoidal category, whenever $\mathbb{G}$ is a quasitriangular quantum group. We recall the explicit construction of the unitary braiding on $\mathfrak{R e p}(\mathbb{G})$ and the monoidal product $\bigotimes_{\mathrm{R}}$ on $\mathfrak{C}^{*} \mathfrak{a l g}(\mathbb{G})$. The latter construction was motivated by [16].

Let $(\alpha, \beta)$ be an R-Heisenberg pair acting on a Hilbert space $\mathcal{L}$. More explicitly, $\alpha, \beta \in \operatorname{Mor}(A, \mathbb{K}(\mathcal{L}))$ and satisfy the commutation relation $\mathrm{W}_{1 \alpha} \mathrm{W}_{2 \beta}=$ $\mathrm{W}_{2 \beta} \mathrm{W}_{1 \alpha} \mathrm{R}_{12}$ in $\mathcal{U}(\hat{A} \otimes \hat{A} \otimes \mathbb{K}(\mathcal{L}))$, where $\mathrm{W}_{1 \alpha}:=\left(\left(\mathrm{id}_{\hat{A}} \otimes \alpha\right) \mathrm{W}\right)_{13}$ and $\mathrm{W}_{1 \beta}:=\left(\left(\mathrm{id}_{\hat{A}} \otimes\right.\right.$ $\beta) \mathrm{W})_{23}$ in $\mathcal{U}(\hat{A} \otimes \hat{A} \otimes \mathbb{K}(\mathcal{L}))$, see [13, Definition 3.1]. Existence of R-Heisenberg pairs is guaranteed by [13, Lemma 3.8].

Suppose $\left(\mathcal{L}_{i}, \mathrm{U}^{i}\right)$ are objects in $\mathfrak{R e p}(\mathbb{G})$ for $i=1,2$. The proof of [13, Theorem 4.1] shows that there is a unique solution $Z \in \mathcal{U}\left(\mathcal{L}_{1} \otimes \mathcal{L}_{2}\right)$, independent of the choice of the R-Heisenberg pair $(\alpha, \beta)$, of the following equation

$$
\mathrm{U}_{1 \alpha}^{1} \mathrm{U}_{2 \beta}^{2} Z_{12}=\mathrm{U}_{2 \beta}^{2} \mathrm{U}_{1 \alpha}^{1} \quad \text { in } \mathcal{U}\left(\mathcal{L}_{1} \otimes \mathcal{L}_{2} \otimes \mathcal{L}\right)
$$

The braiding unitary is defined by $\mathcal{L}_{1} \chi^{\mathcal{L}_{2}}:=Z \circ \Sigma \in \operatorname{Hom}^{\mathbb{G}}\left(\mathrm{U}^{2} \oplus \mathrm{U}^{1}, \mathrm{U}^{1} \oplus \mathrm{U}^{2}\right)$, see [14, Equation 3.2].

Suppose $\left(C_{i}, \delta_{i}\right)$ are objects in $\mathfrak{C}^{*} \mathfrak{a l g}(\mathbb{G})$ and $\left(\mathrm{U}^{i}, \varphi_{i}\right)$ are faithful covariant representations of $\left(C_{i}, \delta_{i}, \mathbb{G}\right)$ on $\mathcal{L}_{i}$ for $i=1,2$. Clearly, $\left(\mathcal{L}_{i}, \mathrm{U}^{i}\right)$ are objects in $\mathfrak{R e p}(\mathbb{G})$ for $i=1,2$ and let ${ }^{\mathcal{L}_{1}} \chi^{\mathcal{L}_{2}}$ be the unitary braiding. Define $j_{i} \in \operatorname{Mor}\left(C_{i}, \mathbb{K}\left(\mathcal{L}_{1} \otimes \mathcal{L}_{2}\right)\right)$ by

$$
j_{1}\left(c_{1}\right):=\varphi_{1}\left(c_{1}\right) \otimes 1_{\mathcal{L}_{2}}, \quad j_{2}\left(c_{2}\right):={ }^{\mathcal{L}_{1}} \chi^{\mathcal{L}_{2}}\left(\varphi_{2}\left(c_{2}\right) \otimes 1_{\mathcal{L}_{1}}\right)^{\mathcal{L}_{1} \chi^{\mathcal{L}_{2}}}{ }^{*}
$$

for all $c_{i} \in C_{i}$ and $i=1,2$. Then $C_{1} \otimes_{\mathrm{R}} C_{2}:=j_{1}\left(C_{1}\right) j_{2}\left(C_{2}\right) \subseteq \mathbb{B}\left(\mathcal{L}_{1} \otimes \mathcal{L}_{2}\right)$ is a $\mathrm{C}^{*}$-algebra [13, Theorem 4.6] and $\delta_{1} \bowtie \delta_{2}\left(j_{1}\left(c_{1}\right) j_{2}\left(c_{2}\right)\right):=\left(j_{1} \otimes \mathrm{id}_{A}\right) \delta_{1}\left(c_{1}\right)\left(j_{2} \otimes\right.$ $\left.\operatorname{id}_{A}\right) \delta_{2}\left(c_{2}\right)$ defines an action of $\mathbb{G}$ on $C_{1} \otimes_{\mathrm{R}} C_{2}$ [14, Proposition 4.1]. Let $\left(C_{i}^{\prime}, \delta_{i}^{\prime}\right)$ be objects in $\mathfrak{C}^{*} \mathfrak{a l g}(\mathbb{G})$ and $f_{i} \in \operatorname{Mor}^{\mathbb{G}}\left(C_{i}, C_{i}^{\prime}\right)$ for $i=1,2$. The monoidal product $f_{1} \bigotimes_{\mathrm{R}}$ $f_{2} \in \operatorname{Mor}\left(C_{1} \otimes_{\mathrm{R}} C_{2}, C_{1}^{\prime} \otimes_{\mathrm{R}} C_{2}^{\prime}\right)$ in $\mathfrak{C}^{*} \mathfrak{a l g}(\mathbb{G})$ is defined by

$$
\left(f_{1} \otimes_{\mathrm{R}} f_{2}\right) \circ j_{1}=j_{1}^{\prime} \circ f_{1}, \quad\left(f_{1} \otimes_{\mathrm{R}} f_{2}\right) \circ j_{2}=j_{2}^{\prime} \circ f_{2},
$$

where $j_{i}^{\prime} \in \operatorname{Mor}\left(C_{i}^{\prime}, C_{1}^{\prime} \otimes_{\mathrm{R}} C_{2}^{\prime}\right)$ are the canonical morphisms for $i=1,2$.

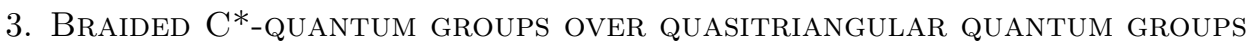

Let $\mathbb{G}=\left(A, \Delta_{A}\right)$ be a quasitriangular quantum group with an $R$-matrix $\mathrm{R} \in$ $\mathcal{U}(\hat{A} \otimes \hat{A})$.

Definition 3.1. Let $(\mathcal{L}, \mathrm{U})$ be an object of $\mathfrak{R e p}(\mathbb{G})$. Let ${ }^{\mathcal{L}}{ }^{\mathcal{L}}=Z \circ \Sigma \in \operatorname{Hom}^{\mathbb{G}}(\mathrm{U} \oplus$ $\mathrm{U}, \mathrm{U} \oplus \mathrm{U})$ be the braiding unitary. A unitary $\mathbb{F} \in \mathcal{U}(\mathcal{L} \otimes \mathcal{L})$ is said to be a braided multiplicative unitary over $\mathbb{G}$ relative to $(\mathrm{U}, \mathrm{R})$ if

(1) $\mathbb{F} \in \operatorname{Hom}^{\mathbb{G}}(\mathrm{U} \oplus \mathrm{U}, \mathrm{U} \oplus \mathrm{U})$ :

$$
\mathbb{F}_{12} \mathrm{U}_{13} \mathrm{U}_{23}=\mathrm{U}_{13} \mathrm{U}_{23} \mathbb{F}_{12} \quad \text { in } \mathcal{U}(\mathbb{K}(\mathcal{L} \otimes \mathcal{L}) \otimes A) ;
$$

(2) $\mathbb{F}$ satisfies the braided pentagon equation:

$$
\mathbb{F}_{23} \mathbb{F}_{12}=\mathbb{F}_{12}\left(\mathcal{L}_{\chi^{\mathcal{L}}}{ }_{23}\right) \mathbb{F}_{12}\left(\mathcal{L}_{\chi^{\mathcal{L}}}{ }_{23}\right)^{*} \mathbb{F}_{23} \quad \text { in } \mathcal{U}(\mathcal{L} \otimes \mathcal{L} \otimes \mathcal{L})
$$

Recall the bounded coinverse $R_{A}$ of $\mathbb{G}$, which is an involutive normal antiautomorphism of $A$, as in [27, Theorem $1.5(4)]$. Then $\mathrm{U}^{c}:=\mathrm{U}^{\mathrm{T} \otimes R_{A}} \in \mathcal{U}(\mathbb{K}(\overline{\mathcal{L}}) \otimes A)$ is the contragradient representation of $\mathrm{U}$, where $\mathrm{T}$ is the transposition defined by $T^{\top} \bar{x}:=\overline{T^{*} x}$ for all $T \in \mathbb{B}(\mathcal{L})$ (see [21, Definition 11]). There is a unique element $\widetilde{Z} \in \mathcal{U}(\overline{\mathcal{L}} \otimes \mathcal{L})$ satisfying (2.12) for the representations $\left(\mathrm{U}^{c}, \mathrm{U}\right)$. 
Suppose $\mathbb{G}$ is constructed from a manageable multiplicative unitary $\mathbb{W} \in \mathcal{U}(\mathcal{H} \otimes$ $\mathcal{H})$ and $Q$ be the strictly positive operator acting on $\mathcal{H}$ appearing in (2.2). Let $\pi \in$ $\operatorname{Mor}(A, \mathbb{K}(\mathcal{H}))$ be the embedding in (2.3) and let $\mathbb{U}:=\left(\operatorname{id}_{\mathbb{K}(\mathcal{L})} \otimes \pi\right) \mathrm{U} \in \mathcal{U}(\mathcal{L} \otimes \mathcal{H})$.

Definition 3.4. A braided multiplicative unitary $\mathbb{F} \in \mathcal{U}(\mathcal{L} \otimes \mathcal{L})$ over $\mathbb{G}$ relative to $(\mathbb{U}, \mathrm{R})$ is said to be manageable if there is a strictly positive operator $Q_{\mathcal{L}}$ on $\mathcal{L}$ and a unitary $\widetilde{\mathbb{F}} \in \mathcal{U}(\overline{\mathcal{L}} \otimes \mathcal{L})$ such that

$$
\mathbb{F}\left(Q_{\mathcal{L}} \otimes Q_{\mathcal{L}}\right) \mathbb{F}^{*}=Q_{\mathcal{L}} \otimes Q_{\mathcal{L}}, \quad \mathbb{U}\left(Q_{\mathcal{L}} \otimes Q\right) \mathbb{U}^{*}=Q_{\mathcal{L}} \otimes Q
$$

and

$$
\left\langle x \otimes u\left|Z^{*} \mathbb{F}\right| y \otimes v\right\rangle=\left\langle\bar{y} \otimes Q_{\mathcal{L}} u\left|\widetilde{\mathbb{F}} \widetilde{Z}^{*}\right| \bar{x} \otimes Q_{\mathcal{L}}^{-1} v\right\rangle
$$

for all $x, y \in \mathcal{L}, u \in \mathcal{D}\left(Q_{\mathcal{L}}\right), v \in \mathcal{D}\left(Q_{\mathcal{L}}^{-1}\right)$.

The main result of this section is the construction of braided $\mathrm{C}^{*}$-quantum groups from manageable braided multiplicative unitaries in $\mathfrak{R e p}(\mathbb{G})$.

Theorem 3.6. Let $\mathbb{F} \in \mathcal{U}(\mathcal{L} \otimes \mathcal{L})$ be a manageable braided multiplicative unitary over a regular quantum group $\mathbb{G}=\left(A, \Delta_{A}\right)$ relative to $(\mathbb{U}, \mathrm{R})$. Let

$$
B:=\left\{\left(\omega \otimes \operatorname{id}_{\mathbb{K}(\mathcal{L})}\right) \mathbb{F} \mid \omega \in \mathbb{B}(\mathcal{L})_{*}\right\}^{\text {-closed linear span }} \subset \mathbb{B}(\mathcal{L}) .
$$

Then

(1) $B$ is a nondegenerate, separable $\mathrm{C}^{*}$-subalgebra of $\mathbb{B}(\mathcal{L})$;

(2) Define $\beta(b):=\mathrm{U}\left(b \otimes 1_{A}\right) \mathrm{U}^{*}$ for all $b \in B$. Then $\beta \in \operatorname{Mor}(B, B \otimes A)$ and $(B, \beta)$ is an object of $\mathfrak{C}^{*} \mathfrak{a l g}(\mathbb{G})$;

(3) $\mathbb{F} \in \mathcal{U}(\mathbb{K}(\mathcal{L}) \otimes B)$;

Consider the twisted tensor product $B \nabla_{\mathrm{R}} B$. Suppose $j_{1}, j_{2} \in \operatorname{Mor}^{\mathbb{G}}\left(B, B \bigotimes_{\mathrm{R}} B\right)$ are the canonical elements.

(4) There exists a unique $\Delta_{B} \in \operatorname{Mor}^{\mathbb{G}}\left(B, B \bigotimes_{\mathrm{R}} B\right)$ characterised by

$$
\left(\operatorname{id}_{\mathbb{K}(\mathcal{L})} \otimes \Delta_{B}\right) \mathbb{F}=\left(\left(\operatorname{id}_{\mathbb{K}(\mathcal{L})} \otimes j_{1}\right) \mathbb{F}\right)\left(\left(\operatorname{id}_{\mathbb{K}(\mathcal{L})} \otimes j_{2}\right) \mathbb{F}\right)
$$

Moreover, $\Delta_{B}$ is coassociative: $\left(\Delta_{B} \bigotimes_{R} \operatorname{id}_{B}\right) \circ \Delta_{B}=\left(\operatorname{id}_{B} \bigotimes_{\mathrm{R}} \Delta_{B}\right) \circ \Delta_{B}$ and satisfies the cancellation conditions: $\Delta_{B}(B) j_{1}(B)=B \otimes_{\mathrm{R}} B=\Delta_{B}(B) j_{2}(B)$. The pair $\left(B, \Delta_{B}\right)$ is said to be the braided $C^{*}$-quantum group (over $\mathbb{G}$ ) generated by the braided multiplicative unitary $\mathbb{F}$.

Proof. Let $(\mathcal{L}, \mathrm{V})$ be the object in $\mathfrak{R e p}(\widehat{\mathbb{G}})$ induced by the $R$-matrix $\mathrm{R}$ from $(\mathcal{L}, \mathrm{U})$ in A.3. The functor $\mathcal{F}: \mathfrak{R e p}(\mathbb{G}) \rightarrow \mathcal{Y} \mathcal{D} \mathfrak{R e p}(\mathbb{G})$ in the Proposition A.2 does not change the underlying Hilbert spaces and the morphisms. Since $\mathbb{F} \in \operatorname{Hom}^{\mathbb{G}}(\mathrm{U} \oplus$ $\mathrm{U}, \mathrm{U} \odot \mathrm{U})$ in $\mathfrak{R e p}(\mathbb{G})$ it is also an endomorphism of the object $(\mathcal{L}, \mathrm{U}, \mathrm{V})$ in $\mathcal{Y} \mathcal{D} \mathfrak{R e p}(\mathbb{G})$; hence $\mathbb{F} \in \operatorname{Hom}^{\widehat{\mathbb{G}}}(\mathrm{V} \oplus \mathrm{V}, \mathrm{V} \oplus \mathrm{V})$ in $\mathfrak{R e p}(\widehat{\mathbb{G}})$. This implies that (3.3) remains same in $\mathcal{Y D} \mathfrak{R e p}(\mathbb{G})$. Hence, $\mathbb{F}$ is also a braided multiplicative unitary in the sense of 15. Definition 3.2]. Let $\pi \in \operatorname{Mor}(A, \mathbb{K}(\mathcal{H}))$ and $\hat{\pi} \in \operatorname{Mor}(\hat{A}, \mathbb{K}(\mathcal{H}))$ be the canonical embeddings in (2.3). Define $\mathrm{R}^{\prime}:=(\hat{\pi} \otimes \hat{\pi}) \mathrm{R} \in \mathcal{U}(\mathcal{H} \otimes \mathcal{H})$ and $\mathbb{V}:=\left(\operatorname{id}_{\mathbb{K}(\mathcal{L})} \otimes \hat{\pi}\right) \mathrm{V} \in$ $\mathcal{U}(\mathcal{L} \otimes \mathcal{H})$. Using [12, Equation 33] we obtain a concrete realisation of (A.3) as follows

$$
\mathrm{R}_{23}^{\prime} \mathbb{U}_{12} \mathrm{R}_{23}^{\prime *}=\mathbb{U}_{12} \mathbb{V}_{13} \quad \text { in } \mathcal{U}(\mathcal{L} \otimes \mathcal{H} \otimes \mathcal{H})
$$

Manageability of $\mathbb{F}$ implies $\mathbb{U}$ commutes with $Q_{\mathcal{L}} \otimes Q$. Since $\mathrm{R}^{\prime}$ is a bicharacter it commutes with $Q \otimes Q$ by [12, Proposition 3.10]. Therefore, $\mathbb{V}_{13}$ commutes with $Q_{\mathcal{L}} \otimes Q \otimes Q$ which implies that $\mathbb{V}$ commutes with $Q_{\mathcal{L}} \otimes Q$. Thus $\mathbb{F}$ is also manageable in the sense of [15, Definition 3.5]. Hence [19, Theorem 5.1] completes the proof. In fact, the conclusions $(1)-(3)$ and, assuming the existence of $\Delta_{B}$ characterised by (3.8), the cancellation conditions in (4) follow immediately. Define $\Delta_{B}(b):=\mathbb{F}\left(b \otimes 1_{\mathcal{L}}\right) \mathbb{F}^{*}$ for all $b \in B$. Observe that $(B \hookrightarrow \mathbb{B}(\mathcal{L}), \mathrm{U})$ is a faithful covariant representation of $(B, \beta, \mathbb{G})$ on $\mathcal{L}$. Hence, we use the braiding operator $\rtimes$ in 
Definition 3.1 to define the canonical embeddings $j_{1}, j_{2} \in \operatorname{Mor}^{\mathbb{G}}\left(B, B \nabla_{\mathrm{R}} B\right)$ in (2.13). Then braided pentagon equation (3.3) and (1) ensures that $\Delta_{B}: B \rightarrow \mathcal{M}\left(B \otimes_{\mathrm{R}} B\right)$ is a $\mathbb{G}$-equivariant ${ }^{*}$-homomorphism, satisfies (3.8) and is coassociative. Finally, $\Delta_{B}(B)\left(B \otimes_{\mathrm{R}} B\right)=\Delta_{B}(B) j_{1}(B) j_{2}(B)=\left(B \otimes_{\mathrm{R}} B\right) j_{2}(B)=B \otimes_{\mathrm{R}} B$ shows that $\Delta_{B}$ is nondegenerate.

\section{Braided multiplicative unitary of $\mathrm{E}_{q}(2)$ Groups}

Let $\left\{e_{p}\right\}_{p \in \mathbb{Z}}$ be an orthonormal basis of $\mathcal{H}=\ell^{2}(\mathbb{Z})$. The operator $\hat{N} e_{p}:=p e_{p}$ is an unbounded densely defined self adjoint operator with $\operatorname{Sp}(\hat{N})=\mathbb{Z}$ and generates the $\mathrm{C}^{*}$-algebra $\mathrm{C}_{0}(\mathbb{Z})$. The operator $z e_{p}:=e_{p+1}$ is unitary and generates the $\mathrm{C}^{*}$-algebra $\mathrm{C}(\mathbb{T})$. A simple computation shows that $z$ and $\hat{N}$ satisfy the following commutation relation

$$
z^{*} \hat{N} z=\hat{N}+1_{\mathcal{H}}
$$

Define $\mathbb{W} \in \mathcal{U}(\mathcal{H} \otimes \mathcal{H})$ by

$$
\mathbb{W}:=\left(1_{\mathcal{H}} \otimes z\right)^{\hat{N} \otimes 1_{\mathcal{H}}}=\int_{\mathbb{Z} \times \mathbb{T}} x^{s} d E_{\hat{N}}(s) \otimes d E_{z}(x),
$$

where $d E_{\hat{N}}$ and $d E_{z}$ are the spectral measures of $\hat{N}$ and $z$, respectively. The commutation relation (4.1) gives the action of $\mathbb{W}$ on the orthonormal basis $\left\{e_{k} \otimes\right.$ $\left.e_{l}\right\}_{k, l \in \mathbb{Z}}$ by $e_{k} \otimes e_{l} \rightarrow e_{k} \otimes e_{l+k}$. It is easy to verify that $\mathbb{W}$ is a manageable multiplicative unitary with $Q=\operatorname{id}_{\mathcal{H}}$ and $\widetilde{\mathbb{W}}\left(\bar{e}_{k} \otimes e_{l}\right):=\bar{e}_{k} \otimes e_{l+k} . \mathbb{W}$ generates $\mathbb{T}$ :

$$
\begin{aligned}
\mathrm{C}(\mathbb{T}) & =\left\{\left(\omega \otimes \mathrm{id}_{\mathbb{K}(\mathcal{H})}\right) \mathbb{W} \mid \omega \in \mathbb{B}(\mathcal{H})_{*}\right\}^{\text {-closed linear span }}, \\
\Delta_{\mathrm{C}(\mathbb{T})}(z) & =\mathbb{W}\left(z \otimes 1_{\mathcal{H}}\right) \mathbb{W}^{*}=z \otimes z .
\end{aligned}
$$

The dual of $\mathbb{T}$ is $\mathbb{Z}$ with the comultiplication map $\Delta_{\mathrm{C}_{0}(\mathbb{Z})}(\hat{N})=\hat{N} \otimes 1_{\mathrm{C}_{0}(\mathbb{Z})}+1_{\mathrm{C}_{0}(\mathbb{Z})} \otimes$ $\hat{N}$.

Recall the $R$-matrix $\mathrm{R}: \mathbb{Z} \times \mathbb{Z} \rightarrow \mathbb{T}$ defined in (1.4). Then $\mathbb{T}$ is a quasitriangular with respect to $\mathrm{R}$ and $\mathfrak{R e p}(\mathbb{T})$ a unitarily braided monoidal category.

Let $\mathcal{L}:=\mathcal{H} \otimes \mathcal{H}$ and fix an orthonormal basis $\left\{e_{i, j}:=e_{i} \otimes e_{j}\right\}_{i, j \in \mathbb{Z}}$ of $\mathcal{L}$. Define $\mathbb{U}:=1_{\mathcal{H}} \otimes \mathbb{W} \in \mathcal{U}\left(\mathcal{H}^{\otimes 3}\right) \cong \mathcal{U}(\mathcal{L} \otimes \mathcal{H})$. Clearly, $\mathbb{U}\left(e_{i, j} \otimes e_{p}\right)=e_{i, j} \otimes e_{p+j}$ and denote $\mathbb{U}$ by $U$ while viewed as an element of $\mathcal{U}(\mathbb{K}(\mathcal{L}) \otimes \mathrm{C}(\mathbb{T}))$. Also, the first equation in (2.4) shows that $U$ is a representation of $\mathbb{T}$ on $\mathcal{L}$. Hence $(\mathcal{L}, U)$ is an object of $\mathfrak{R e p}(\mathbb{T})$.

Define $\alpha, \beta \in \operatorname{Mor}(\mathrm{C}(\mathbb{T}), \mathbb{K}(\mathcal{H}))$ by $\alpha(z):=z$ and $\beta(z):=\widetilde{V}$, where $\widetilde{V}$ is the unitary operator defined by $\widetilde{V} e_{p}=\zeta^{-p} e_{p}$, respectively. Then $(\alpha, \beta)$ is an R-Heisenberg pair on $\mathcal{H}$ because $z$ and $\widetilde{V}$ commute up to $\zeta$ :

$$
z \widetilde{V} e_{p}=\zeta^{-p} z e_{p}=\zeta^{-p} e_{p+1}=\zeta \widetilde{V} e_{p+1}=\zeta \widetilde{V} z e_{p}
$$

In fact, $z$ and $\widetilde{V}$ generate noncommutative two torus. Using these we compute the unitary $Z \in \mathcal{U}(\mathcal{L} \otimes \mathcal{L})$ in (2.12). Equivalently, $Z$ is uniquely defined by

$$
Z_{12}=\mathrm{U}_{2 \beta}^{*} \mathrm{U}_{1 \alpha}^{*} \mathrm{U}_{2 \beta} \mathrm{U}_{1 \alpha} \quad \text { in } \mathcal{U}(\mathcal{L} \otimes \mathcal{L} \otimes \mathcal{H}),
$$

where $\mathrm{U}_{1 \alpha}=\mathrm{U}$ and $\mathrm{U}_{1 \beta}=\left(1_{\mathcal{H}} \otimes 1_{\mathcal{H}} \otimes \widetilde{V}\right)^{\left(1_{\mathcal{H}} \otimes \hat{N} \otimes 1_{\mathcal{H}}\right)}$. Clearly, on the basis elements we have $\mathrm{U}_{1 \beta}\left(e_{k, l} \otimes e_{p}\right)=\zeta^{-p l} e_{k, l} \otimes e_{p}$. Therefore,

$$
\begin{aligned}
\mathrm{U}_{2 \beta}^{*} \mathrm{U}_{1 \alpha}^{*} \mathrm{U}_{2 \beta} \mathrm{U}_{1 \alpha}\left(e_{i, j} \otimes e_{k, l} \otimes e_{p}\right) & =\zeta^{-(p+j) l} \mathrm{U}_{2 \beta}^{*} \mathrm{U}_{1 \alpha}^{*}\left(e_{i, j} \otimes e_{k, l} \otimes e_{p+j}\right) \\
& =\zeta^{-(p+j) l+p l} e_{i, j} \otimes e_{k, l} \otimes e_{p}=\zeta^{-j l} e_{i, j} \otimes e_{k, l} \otimes e_{p} .
\end{aligned}
$$

Hence the the unitary braiding operator $x:=Z \circ \Sigma \in \mathcal{U}(\mathcal{L} \otimes \mathcal{L})$ is given by

$$
Z\left(e_{i, j} \otimes e_{k, l}\right)=\zeta^{-j l} e_{i, j} \otimes e_{k, l}, \quad \rtimes e_{i, j} \otimes e_{k, l}=\zeta^{-j l} e_{k, l} \otimes e_{i, j} .
$$


Define a closed operator $X=|X| \Phi_{X}$ on $\mathcal{L} \otimes \mathcal{L}$ by

$$
\begin{aligned}
|X| e_{i, j} \otimes e_{k, l} & =|q|^{k-i+1} e_{i, j} \otimes e_{k, l}, \\
\Phi_{X} e_{i, j} \otimes e_{k, l} & =\zeta^{-j} \Phi_{q}^{k-i+1} e_{i-1, j-1} \otimes e_{k-1, l+1}, \\
X e_{i, j} \otimes e_{k, l} & =\zeta^{-j} q^{k-i+1} e_{i-1, j-1} \otimes e_{k-1, l+1} .
\end{aligned}
$$

Then $X$ is a normal operator because $|X|$ commutes with its phase $\Phi_{X}$ in the polar decomposition and $\operatorname{Sp}(X)=\overline{\mathbb{C}}^{|q|}$.

Recall that the operator $n$, defined by $n e_{i, j}=q^{i} e_{i, j+1}$, is closed and injective. Hence it is invertible and on the standard basis elements we have $n^{-1} e_{i, j}=q^{-i} e_{i, j-1}$. Define $P \in \mathcal{U}(\mathcal{L})$ by

$$
P e_{i, j}=\zeta^{-j} e_{i, j}
$$

Then the following computation shows $X=n^{-1} v P \otimes v n$ :

$\left(n^{-1} v P \otimes v n\right) e_{i, j} \otimes e_{k, l}=\zeta^{-j} q^{k}\left(n^{-1} \otimes v\right) e_{i-1, j} \otimes e_{k, l+1}=\zeta^{-j} q^{k-i+1} e_{i-1, j-1} \otimes e_{k-1, l+1}$.

The quantum exponential function $F_{|q|}: \overline{\mathbb{C}}^{|q|} \rightarrow \mathbb{T}$ is defined in [24, Equation 1.2] by

$$
F_{|q|}(z)= \begin{cases}\prod_{k=0}^{\infty} \frac{1+|q|^{2 k} \bar{z}}{1+|q|^{2 k} z} & \text { if } z \in \overline{\mathbb{C}}^{|q|} \backslash\left\{-|q|^{-2 k} \mid k=0,1, \cdots\right\} \\ -1 & \text { otherwise }\end{cases}
$$

Since $F_{|q|}$ is a unitary multiplier of $\mathrm{C}_{0}\left(\overline{\mathbb{C}}^{|q|}\right)$, we observe $F_{|q|}(X) \in \mathcal{U}(\mathcal{L} \otimes \mathcal{L})$.

Theorem 4.6. Let $\mathbb{Y}=\mathbb{W}_{13} \mathbb{W}_{23} \in \mathcal{U}(\mathcal{H} \otimes \mathcal{H} \otimes \mathcal{H} \otimes \mathcal{H})$. Then the operator $\mathbb{F}:=F_{|q|}(X) \mathbb{Y}$ is a manageable braided multiplicative unitary on $\mathcal{L} \otimes \mathcal{L}$ over $\mathbb{T}$ relative to $(\mathrm{U}, \mathrm{R})$.

The rest of this section is devoted to the proof of this theorem.

Lemma 4.7. Define $T(\lambda):=F_{|q|}(\lambda X)$ for all $\lambda \in \overline{\mathbb{C}}^{|q|}$. Then

$$
F_{|q|}(X)_{23} T(\lambda)_{12}=T(\lambda)_{12} F_{|q|}\left(\lambda n^{-1} v P \otimes v^{2} P \otimes v n\right) F_{|q|}(X)_{23} \quad \text { in } \mathcal{U}(\mathcal{L} \otimes \mathcal{L} \otimes \mathcal{L}) .
$$

Proof. Clearly, the equality holds for $\lambda=0$. Therefore, we fix a nonzero element $\lambda \in \overline{\mathbb{C}}^{|q|}$. The operators $R=\lambda n^{-1} v P \otimes v n \otimes 1_{\mathcal{L}}=\lambda X_{12}$ and $S=\lambda n^{-1} v P \otimes$ $v^{2} P \otimes v n=\lambda\left(1_{\mathcal{L}} \otimes v^{2} P \otimes 1_{\mathcal{L}}\right) X_{13}$ are normal, each pair of operators $(|R|,|S|)$ and $\left(\Phi_{R}, \Phi_{S}\right)$ strongly commute and

$$
\Phi_{R}|S| \Phi_{R}^{*}=|q|^{-1}|S|, \quad \Phi_{S}|R| \Phi_{S}^{*}=|q||R|, \quad \operatorname{Sp}(R), \operatorname{Sp}(S)=\overline{\mathbb{C}}^{|q|} .
$$

Also $R^{-1} S=1_{\mathcal{L}} \otimes n^{-1} v P \otimes v n=X_{23}$ is normal with spectrum $\overline{\mathbb{C}}|q|$. Then [24, theorem 2.1-2.2] show that $R \dot{+} S$ is normal with spectrum $\overline{\mathbb{C}}^{|q|}$ and

$$
F_{|q|}\left(R^{-1} S\right) R F_{|q|}\left(R^{-1} S\right)^{*}=R \dot{+} S .
$$

Since the functional calculus is compatible with conjugation by unitaries, 24, Theorem 3.1] implies

$$
\begin{aligned}
F_{|q|}\left(R^{-1} S\right) F_{|q|}(R) F_{|q|}\left(R^{-1} S\right)^{*} & =F_{|q|}\left(F_{|q|}\left(R^{-1} S\right) R F_{|q|}\left(R^{-1} S\right)^{*}\right) \\
& =F_{|q|}(R+S)=F_{|q|}(R) F_{|q|}(S) .
\end{aligned}
$$

For $\lambda \in \overline{\mathbb{C}}^{|q|}$ define $\mathbb{F}^{\lambda}:=F_{|q|}(\lambda X) \mathbb{Y} \in \mathcal{U}(\mathcal{L} \otimes \mathcal{L})$.

Proposition 4.8. The family of unitaries $\left\{\mathbb{F}^{\lambda}\right\}_{\lambda \in \overline{\mathbb{C}}^{|q|}}$ commute with $\mathbb{U} \oplus \mathbb{U}$ and satisfy a variant of the braided pentagon equation (13.3):

$$
\mathbb{F}_{23} \mathbb{F}_{12}^{\lambda}=\mathbb{F}_{12}^{\lambda} \succ_{23} \mathbb{F}_{12}^{\lambda} \succ_{23}^{*} \mathbb{F}_{23} \quad \text { in } \mathcal{U}(\mathcal{L} \otimes \mathcal{L} \otimes \mathcal{L})
$$


Proof. The unitaries $\mathbb{W}_{25} \mathbb{W}_{45}$ and $\mathbb{W}_{13} \mathbb{W}_{23}$ commute in $\mathcal{U}\left(\mathcal{H}^{\otimes 5}\right)$. Identifying $\mathcal{L}=$ $\mathcal{H} \otimes \mathcal{H}$ and $\mathcal{L} \otimes \mathcal{L} \otimes \mathcal{H}=\mathcal{H}^{\otimes 5}$ we get $\mathbb{U} \oplus \mathbb{U}$ and $\mathbb{Y}_{12}$ commutes in $\mathcal{U}(\mathcal{L} \otimes \mathcal{L} \otimes \mathcal{H})$. Moreover $\mathbb{U} \oplus \mathbb{U}\left(e_{i, j} \otimes e_{k, l} \otimes e_{p}\right)=e_{i, j} \otimes e_{k, l} \otimes e_{p+j+l}$ shows the operators $|X|_{12}$ and $\left(\Phi_{X}\right)_{12}$ defined in (4.4) commute with $\mathbb{U} \oplus \mathbb{U}$. Therefore $\mathbb{U} \oplus \mathbb{U}$ commutes with $\lambda X_{12}$ for all $\lambda \in \overline{\mathbb{C}}^{|q|}$. Therefore, $\mathbb{U} \oplus \mathbb{U}$ commutes with $F_{|q|}(\lambda X)_{12}$ and consequently with $\mathbb{F}_{12}^{\lambda}$ in $\mathcal{U}(\mathcal{L} \otimes \mathcal{L} \otimes \mathcal{H})$.

The action of $v n$ and $\mathbb{Y}=\mathbb{W}_{13} \mathbb{W}_{23}$ on the basis elements of $\mathcal{L}$ and $\mathcal{L} \otimes \mathcal{L}$ are given by $e_{i, j} \rightarrow q^{i} e_{i-1, j+1}$ and $e_{i, j} \otimes e_{k, l} \rightarrow e_{i, j} \otimes e_{k+i+j, l}$, respectively. Therefore,

$$
\begin{aligned}
\mathbb{Y}\left(v n \otimes 1_{\mathcal{L}}\right) \mathbb{Y}^{*} e_{i, j} \otimes e_{k, l} & =\mathbb{Y}\left(v n \otimes 1_{\mathcal{L}}\right) e_{i, j} \otimes e_{k-i-j, l} \\
& =q^{i} \mathbb{Y} e_{i-1, j+1} \otimes e_{k-i-j, l} \\
& =q^{i} e_{i-1, j+1} \otimes e_{k, l}=\left(v n \otimes 1_{\mathcal{L}}\right) e_{i, j} \otimes e_{k, l}
\end{aligned}
$$

implies that $\mathbb{Y}$ commutes with $v n \otimes 1_{\mathcal{L}}$. This implies that 4.9 is equivalent to

$$
F_{|q|}(X)_{23} T(\lambda)_{12} \mathbb{Y}_{23} \mathbb{Y}_{12} \mathbb{Y}_{23}^{*} F_{|q|}(X)_{23}^{*}=T(\lambda)_{12} \mathbb{Y}_{12} Z_{23} T(\lambda)_{13} \mathbb{Y}_{13} Z_{23}^{*}
$$

where $\{T(\lambda)\}_{\lambda \in \overline{\mathbb{C}}^{|q|}}$ is the family of unitary operators defined in Lemma 4.7

Now $\mathbb{Y}$ is a multiplicative unitary because $\mathbb{Y}_{23} \mathbb{Y}_{12}\left(e_{i, j} \otimes e_{k, l} \otimes e_{s, t}\right)=e_{i, j} \otimes$ $e_{k+i+j, l} \otimes e_{s+k+i+j+l, t}=\mathbb{Y}_{12} \mathbb{Y}_{13} \mathbb{Y}_{23}\left(e_{i, j} \otimes e_{k, l} \otimes e_{s, t}\right)$. This simplifies the last equation to

$$
F_{|q|}(X)_{23} T(\lambda)_{12} \mathbb{Y}_{12} \mathbb{Y}_{13} F_{|q|}(X)_{23}^{*}=T(\lambda)_{12} \mathbb{Y}_{12} Z_{23} T(\lambda)_{13} \mathbb{Y}_{13} Z_{23}^{*} .
$$

Using (4.4) we compute

$$
\begin{aligned}
& \mathbb{Y}_{13}^{*} \mathbb{Y}_{12}^{*} X_{23} \mathbb{Y}_{12} \mathbb{Y}_{13}\left(e_{i, j} \otimes e_{k, l} \otimes e_{s, t}\right) \\
& =\mathbb{Y}_{13}^{*} \mathbb{Y}_{12}^{*} X_{23}\left(e_{i, j} \otimes e_{k+i+j, l} \otimes e_{s+i+j, t}\right) \\
& =\zeta^{-l} q^{(s+i+j)-(k+i+j)+1} \mathbb{Y}_{13}^{*} \mathbb{Y}_{12}^{*}\left(e_{i, j} \otimes e_{k+i+j-1, l-1} \otimes e_{s+i+j-1, t+1}\right) \\
& =\zeta^{-l} q^{s-k+1} e_{i, j} \otimes e_{k-1, l-1} \otimes e_{s-1, t+1}=X_{23}\left(e_{i, j} \otimes e_{k, l} \otimes e_{s, t}\right) .
\end{aligned}
$$

Therefore, $\mathbb{Y}_{12} \mathbb{Y}_{13}$ commutes with $F_{|q|}(X)_{23}$ and this implies

$$
F_{|q|}(X)_{23} T(\lambda)_{12} F_{|q|}(X)_{23}^{*} \mathbb{Y}_{12} \mathbb{Y}_{13}=T(\lambda)_{12} \mathbb{Y}_{12} Z_{23} T(\lambda)_{13} \mathbb{Y}_{13} Z_{23}^{*}
$$

Next we compute

$$
\begin{aligned}
& \mathbb{Y}_{12} Z_{23} X_{13} Z_{23}^{*} \mathbb{Y}_{12}^{*}\left(e_{i, j} \otimes e_{k, l} \otimes e_{s, t}\right) \\
& =\zeta^{l t} \mathbb{Y}_{12} Z_{23} X_{13}\left(e_{i, j} \otimes e_{k-i-j, l} \otimes e_{s, t}\right) \\
& =\zeta^{l t} \zeta^{-j} q^{s-i+1} \mathbb{Y}_{12} Z_{23}\left(e_{i-1, j-1} \otimes e_{k-i-j, l} \otimes e_{s-1, t+1}\right) \\
& =\zeta^{l t-j-l(t+1)} q^{s-i+1} e_{i-1, j-1} \otimes e_{k-2, l} \otimes e_{s-1, t+1} \\
& =X_{13}\left(1_{\mathcal{L}} \otimes v^{2} P \otimes 1_{\mathcal{L}}\right)\left(e_{i, j} \otimes e_{k, l} \otimes e_{s, t}\right) .
\end{aligned}
$$

This implies

$F_{|q|}(X)_{23} T(\lambda)_{12} F_{|q|}(X)_{23}^{*} \mathbb{Y}_{12} \mathbb{Y}_{13}=T(\lambda)_{12} F_{|q|}\left(\lambda n^{-1} v P \otimes v^{2} P \otimes v n\right) \mathbb{Y}_{12} Z_{23} \mathbb{Y}_{13} Z_{23}^{*}$.

Finally, observe that $\mathbb{Y}_{13}$ commutes with $Z_{23}$ and then the last equation follows from Lemma 4.7.

In particular, for $\lambda=1$ the Proposition 4.8 shows that $\mathbb{F}$ is a braided multiplicative unitary over $\mathbb{T}$ with respect to $(\mathbb{U}, R)$.

It remains to show that $\mathbb{F}$ is manageable. We start with the description of the operator $\widetilde{Z}$ appearing in the definition 3.4. The contragradient $\mathrm{U}^{c}=\mathrm{U}^{\mathrm{T} \otimes R}$ of $\mathrm{U}$, where $R(z):=z^{*}$ is the bounded coinverse of $\mathbb{T}$, acts on the basis elements $\overline{e_{i, j}} \otimes e_{p}$ as $\mathbb{U}^{c}\left(\overline{e_{i, j}} \otimes e_{p}\right)=\overline{e_{i, j}} \otimes e_{p-j}$. A similar calculation for $\widetilde{Z}$ like that of $Z$ yields

$$
\widetilde{Z} \overline{e_{i, j}} \otimes e_{k, l}=\zeta^{j l} \overline{e_{i, j}} \otimes e_{k, l} .
$$


Next we define the operator $Q_{\mathcal{L}}$ required by definition 3.4

$$
Q_{\mathcal{L}} e_{i, j}=|q|^{j} e_{i, j}
$$

This is a strictly positive operator on $\mathcal{H}$ with spectrum $|q|^{\mathbb{Z}} \cup\{0\}$. A simple calculation shows that $\mathbb{Y}$ and $X$ commute with $Q_{\mathcal{L}} \otimes Q_{\mathcal{L}}$ and therefore $\mathbb{F}$ commutes with $Q_{\mathcal{L}} \otimes Q_{\mathcal{L}}$. Also the unitary $\mathbb{U}$ leaves the first factor of the standard basis vector unchanged and $Q=\mathrm{id}_{\mathcal{H}}$ implies $\mathbb{U}$ commutes with $Q_{\mathcal{L}} \otimes Q$.

Finally, we need a unitary $\widetilde{\mathbb{F}} \in \mathcal{U}(\overline{\mathcal{L}} \otimes \mathcal{L})$ that satisfies (3.5). It is sufficient to check this if the involved vectors $x, y, u, v$ are standard basis vectors $x=e_{i, j}$, $y=e_{s, t}, u=e_{k, l}$ and $v=e_{a, b}$. Using the explicit formulas for $Z, \widetilde{Z}, \mathbb{Y}$ and $Q$, we rewrite (3.5) as

$$
\zeta^{j l}\left\langle e_{i, j} \otimes e_{k, l}\left|F_{|q|}(X)\right| e_{s, t} \otimes e_{a+s+t, b}\right\rangle=|q|^{l-b} \zeta^{-j b}\left\langle\overline{e_{s, t}} \otimes e_{k, l}|\widetilde{\mathbb{F}}| \overline{e_{i, j}} \otimes e_{a, b}\right\rangle
$$

for all $a, b, i, j, k, l, s, t \in \mathbb{Z}$. To compute the left hand side of (4.11) we shall use the Fourier transform of $F_{|q|}$ restricted on the concentric circles $|z| \in|q|^{\mathbb{Z}}$

$$
F_{|q|}(z)=\sum_{m \in \mathbb{Z}} F_{m}(|z|) \Phi_{z}^{m}
$$

where $z=\Phi_{z}|z|$ and the coefficients $F_{m}\left(|q|^{n}\right)$ for $m, n \in \mathbb{Z}$ are real and satisfies

$$
F_{m}\left(|q|^{n}\right)=(-|q|)^{m} F_{-m}\left(|q|^{n-m}\right),
$$

see 1] or [28, Appendix A].

Now $e_{s, t} \otimes e_{a+s+t, b}$ is an eigenvector of $|X|$ with eigenvalue $|q|^{a+t+1}$ and $\Phi_{X}^{m}$ acts on it by $e_{s, t} \otimes e_{a+s+t, b} \rightarrow \zeta^{-m t+\frac{m(m-1)}{2}} \Phi_{q}^{m(a+t+1)} e_{s-m, t-m} \otimes e_{a+s+t-m, b+m}$.

Thus

$$
\begin{aligned}
& \zeta^{j l}\left\langle e_{i, j} \otimes e_{k, l}\left|F_{|q|}(X)\right| e_{s, t} \otimes e_{a+s+t, b}\right\rangle \\
&=\sum_{m \in \mathbb{Z}} \zeta^{j l}\left\langle e_{i, j} \otimes e_{k, l}\left|\Phi_{X}^{m} F_{m}(|X|)\right| e_{s, t} \otimes e_{a+s+t, b}\right\rangle \\
&=\sum_{m \in \mathbb{Z}} \zeta^{j l-m t+\frac{m(m-1)}{2}} \Phi_{q}^{m(a+t+1)} F_{m}\left(|q|^{a+t+1}\right) \delta_{i, s-m} \delta_{j, t-m} \delta_{k, a+s+t-m} \delta_{l, b+m} \\
&=\zeta^{j l-(l-b) t+\frac{(l-b)(l-b-1)}{2}} \Phi_{q}^{(l-b)(a+t+1)} F_{l-b}\left(|q|^{a+t+1}\right) \delta_{i, s-l+b} \delta_{j, t-l+b} \delta_{k, a+s+t-l+b} \\
&=(-|q|)^{l-b} \zeta^{j l-(l-b) t+\frac{(l-b)(l-b-1)}{2}} \Phi_{q}^{(l-b)(a+t+1)} F_{b-l}\left(|q|^{a+t+1-l+b}\right) \\
& \quad \delta_{i, s-l+b} \delta_{j, t-l+b} \delta_{l-b, a+s+t-k} .
\end{aligned}
$$

The last expression is nonzero if and only if $l-b=s-i=t-j=a+s+t-k$. We also notice that $\zeta=\Phi_{q}^{2}$. These imply that $a+s+t-k=k-a-i-j, \delta_{l-b, a+s+t-k}=$ $\delta_{l-b, k-a-i-j}=\delta_{k+b-l, a+i+j}$ and $\zeta^{j l-(l-b) t+\frac{(l-b)(l-b-1)}{2}} \Phi_{q}^{(l-b)(a+t+1)}=\zeta^{j b} \Phi_{q}^{(b-l)(s-k)}$. Therefore, the left hand side of (4.11) becomes

$$
\begin{aligned}
& \zeta^{j l}\left\langle e_{i, j} \otimes e_{k, l}\left|F_{|q|}(X)\right| e_{s, t} \otimes e_{a+s+t, b}\right\rangle \\
& =(-|q|)^{l-b} \zeta^{j b} \Phi_{q}^{(b-l)(s-k)} F_{b-l}\left(|q|^{k-s+1}\right) \delta_{i, s-l+b} \delta_{j, t-l+b} \delta_{k+b-l, a+i+j} .
\end{aligned}
$$

Now we define an unbounded normal operator $\widetilde{X}=|\widetilde{X}| \Phi_{\widetilde{X}}$ with spectrum $\overline{\mathbb{C}}^{|q|}$

$$
|\widetilde{X}|\left(\overline{e_{i j}} \otimes e_{k l}\right):=|q|^{k-i+1} \overline{e_{i j}} \otimes e_{k l}, \quad \Phi_{\widetilde{X}}\left(\overline{e_{i j}} \otimes e_{k l}\right):=-\Phi_{q}^{k-i} \overline{e_{i+1, j+1}} \otimes e_{k+1, l+1},
$$


and a unitary operator $\widetilde{\mathbb{Y}}\left(\overline{e_{i j}} \otimes e_{k l}\right):=\overline{e_{i j}} \otimes e_{k+i+j, l}$. We show that the unitary $\widetilde{\mathbb{F}}:=F_{|q|}(\widetilde{X})^{*} \widetilde{Z}^{2} \widetilde{\mathbb{Y}}$ satisfies (4.11):

$$
\begin{aligned}
& |q|^{l-b} \zeta^{-j b}\left\langle\overline{e_{s, t}} \otimes e_{k, l}|\widetilde{\mathbb{F}}| \overline{e_{i, j}} \otimes e_{a, b}\right\rangle \\
& =|q|^{l-b} \zeta^{-j b}\left\langle\overline{e_{s, t}} \otimes e_{k, l}\left|F_{|q|}(\widetilde{X})^{*} \widetilde{Z}^{2}\right| \overline{e_{i, j}} \otimes e_{a+i+j, b}\right\rangle \\
& =|q|^{l-b} \zeta^{j b}\left\langle\overline{e_{s, t}} \otimes e_{k, l}\left|F_{|q|}(\widetilde{X})^{*}\right| \overline{e_{i, j}} \otimes e_{a+i+j, b}\right\rangle \\
& =\sum_{m \in \mathbb{Z}}|q|^{l-b} \zeta^{j b}\left\langle\overline{e_{s, t}} \otimes e_{k, l}\left|F_{m}(|\widetilde{X}|)^{*}\left(\Phi_{\widetilde{X}}^{*}\right)^{m}\right| \overline{e_{i, j}} \otimes e_{a+i+j, b}\right\rangle \\
& =\sum_{m \in \mathbb{Z}}|q|^{l-b} \zeta^{j b} F_{m}\left(|q|^{k-s+1}\right)\left\langle\overline{e_{s, t}} \otimes e_{k, l}\left|\left(\Phi_{\widetilde{X}}^{*}\right)^{m}\right| \overline{e_{i, j}} \otimes e_{a+i+j, b}\right\rangle \\
& =\sum_{m \in \mathbb{Z}}|q|^{l-b} \zeta^{j b} F_{m}\left(|q|^{k-s+1}\right)(-1)^{m} \Phi_{q}^{m(s-k)} \delta_{s+m, i} \delta_{t+m, j} \delta_{k+m, a+i+j} \delta_{l+m, b} \\
& =(-|q|)^{l-b} \zeta^{j b} \Phi_{q}^{(b-l)(s-k)} F_{b-l}\left(|q|^{k-s+1}\right) \delta_{i, s-l+b} \delta_{j, t-l+b} \delta_{k+b-l, a+i+j} .
\end{aligned}
$$

\section{Braided $\mathrm{E}_{q}(2)$-Groups AND Bosonisation}

Fix $q \in \mathbb{C}$ with $0<|q|<1$. Now we are going to describe the braided $\mathrm{C}^{*}$-quantum group constructed from the manageable braided multiplicative unitary $\mathbb{F}$ in Theorem 4.6. Hence we shall use all the notations introduced in the previous section.

Recall the $\mathbb{Z}$-action $\alpha$ on $\mathrm{C}_{0}\left(\overline{\mathbb{C}}^{|q|}\right)$ defined by (1.2): $\left(\alpha_{m} f\right)(\lambda):=f\left(q^{m} \lambda\right)$ for $m \in$ $\mathbb{Z}, f \in \mathrm{C}_{0}\left(\overline{\mathbb{C}}^{|q|}\right), \lambda \in \overline{\mathbb{C}}^{|q|}$. Let $\beta$ be the $\mathbb{Z}$-action on $\mathrm{C}_{0}\left(\overline{\mathbb{C}}^{|q|}\right)$ obtained by replacing $q$ by $|q|$ in the definition of $\alpha(1.2)$. Following a similar set of arguments used in [8, Theorem 2.3] we first prove the following result.

Proposition 5.1. The $\mathrm{C}^{*}$-algebras $\mathrm{C}_{0}\left(\overline{\mathbb{C}}^{|q|}\right) \rtimes_{\alpha} \mathbb{Z}$ and $\mathrm{C}_{0}\left(\overline{\mathbb{C}}^{|q|}\right) \rtimes_{\beta} \mathbb{Z}$ are isomorphic.

Proof. Let $q=|q| e^{i \theta}$ be the polar decomposition of $q$. For any element $\lambda \in \overline{\mathbb{C}}^{|q|}$, define

$$
g_{\theta}(\lambda)= \begin{cases}\lambda e^{-i \theta \log _{|q|}|\lambda|} & \lambda \neq 0, \\ 0 & \lambda=0 .\end{cases}
$$

If $\lambda=|q|^{m} e^{i \psi} \neq 0$, then $g_{\theta}(\lambda)=\lambda e^{-i m \theta}$. Thus $g_{\theta}$ is continuous at all nonzero $\lambda \in \overline{\mathbb{C}}^{|q|}$. Let $\left\{\lambda_{k}\right\}$ be a nonzero sequence in $\overline{\mathbb{C}}^{|q|}$ such that $\lambda_{k} \rightarrow 0$ as $k \rightarrow \infty$. Then

$$
g_{\theta}\left(\lambda_{k}\right)=\lambda_{k} e^{-i \theta \log _{|q|}\left|\lambda_{k}\right|} \quad \text { for all }\left|\lambda_{k}\right| \neq 0 .
$$

Since $\left|e^{-i \theta \log _{|q|}\left|\lambda_{k}\right|}\right|$ is bounded for all $\left|\lambda_{k}\right| \neq 0$ we have $g_{\theta}\left(\lambda_{k}\right) \rightarrow 0=g_{\theta}(0)$ as $k \rightarrow$ $\infty$. Hence, $g_{\theta}$ is continuous at all $\lambda \in \overline{\mathbb{C}}^{|q|}$. Also, $g_{\theta}$ separates points of $\overline{\mathbb{C}}^{|q|}$ and $\lim _{\lambda \rightarrow \infty}|g(\lambda)|=+\infty$. Therefore, $g_{\theta}$ generates $\mathrm{C}_{0}\left(\overline{\mathbb{C}}^{|q|}\right)$, see [26, Section 3, Example 2].

Similarly, $g_{-\theta}$ is also continuous and it is the inverse of $g_{\theta}$. So, $g_{\theta}$ is a homeomorphism of $\overline{\mathbb{C}}^{|q|}$. Therefore, $g_{\theta}(n)$ also generates $\mathrm{C}_{0}\left(\overline{\mathbb{C}}^{|q|}\right)$. A simple computation gives

$$
g_{\theta}(q \lambda)=q \lambda e^{-i \theta \log _{|q|}|q \lambda|}=q e^{-i \theta} \lambda e^{-i \theta \log _{|q|}|q \lambda|}=|q| g_{\theta}(\lambda) .
$$

Then using functional calculus and the commutation relation (1.1) we obtain

$$
v g(n) v^{*}=g(q n)=|q| g_{\theta}(n) .
$$

This gives a canonical $\mathbb{Z}$ action, denoted by $\beta$, on $\mathrm{C}_{0}\left(\overline{\mathbb{C}}^{|q|}\right)$ replacing $q$ by $|q|$ in $(1.2)$ and the map $n \mapsto g_{\theta}(n)$ is $\mathbb{Z}$-equivariant; hence it extends to an isomorphism of crossed products $\mathrm{C}_{0}\left(\overline{\mathbb{C}}^{|q|}\right) \rtimes_{\alpha} \mathbb{Z}$ and $\mathrm{C}_{0}\left(\overline{\mathbb{C}}^{|q|}\right) \rtimes_{\beta} \mathbb{Z}$. 
5.1. The underlying $\mathbf{C}^{*}$-algebra. For any closed densely defined operator $T$ acting on a Hilbert space $\mathcal{K}$ the $z$-transform $z_{T} \in \mathbb{B}(\mathcal{K})$ of $T$ is defined by $z_{T}:=$ $T\left(1_{\mathcal{K}}+T^{*} T\right)^{-\frac{1}{2}}$. Moreover, $T$ is affiliated with a $\mathrm{C}^{*}$-algebra $E$, denoted by $T \eta E$, if $z_{T} \in \mathcal{M}(E)$ and $\left(1_{\mathcal{K}}-z_{T}^{*} z_{T}\right)^{\frac{1}{2}} E$ is dense in $E$. If $T \in \mathbb{B}(\mathcal{K})$ and $T \eta E$, then $T \in \mathcal{M}(E)$, see [23, Example 1].

Consider the crossed product $\mathrm{C}^{*}$-algebra $B=\mathrm{C}_{0}\left(\overline{\mathbb{C}}^{|q|}\right) \rtimes_{\alpha} \mathbb{Z}$. Recall that $B$ is generated by $v$ and $n$ in the sense of [26, Definition 3.1]. In particular, this means $v \in \mathcal{M}(B)$ and $n \eta B$. Now $z_{v n}=v z_{n}$ implies $v n \eta B$. Therefore, the operators $v, v n \eta B$. It is also easy to verify that the pair of operators $(v, v n)$ satisfy the conditions of [26, Theorem 3.3]; hence $v$, vn also generate $B$.

Since $v n \eta B$ and $F_{|q|} \in \mathcal{M}\left(\mathrm{C}_{0}\left(\overline{\mathbb{C}}^{|q|}\right)\right)$ we get $n^{-1} v P \otimes v n=X \eta \mathbb{K}(\mathcal{L}) \otimes B$ and consequently $F_{|q|}(X) \in \mathcal{U}(\mathbb{K}(\mathcal{L}) \otimes B)$. Also observe that

$$
\mathbb{Y}=\left(1_{\mathcal{H} \otimes \mathcal{H}} \otimes v^{*}\right)^{\left(\hat{N} \otimes 1_{\mathcal{H}}+1_{\mathcal{H}} \otimes \hat{N}\right) \otimes 1_{\mathcal{L}}} \in \mathcal{U}(\mathcal{H} \otimes \mathcal{H} \otimes \mathcal{L}) \cong \mathcal{U}(\mathcal{L} \otimes \mathcal{L}),
$$

where $\hat{N}$ is the self adjoint densely defined operator acting on $\mathcal{H}$ defined in Section 4 It was already observed in the proof of Proposition 4.8 that $\mathbb{Y}$ is a multiplicative unitary. It is also easy to verify that $\mathbb{Y}$ is manageable with $Q_{\mathcal{L}}=\operatorname{id} \mathrm{d}_{\mathcal{L}}$ and $\widetilde{\mathbb{Y}}\left(\overline{e_{i, j}} \otimes\right.$ $\left.e_{k, l}\right):=\overline{e_{i, j}} \otimes e_{k+i+j, l}$, and also generates $\mathbb{T}$. Thus $\mathbb{Y} \in \mathcal{U}(\mathbb{K}(\mathcal{L}) \otimes \mathrm{C}(\mathbb{T})) \subset \mathcal{U}(\mathbb{K}(\mathcal{L}) \otimes$ $B)$, and consequently $\mathbb{F} \in \mathcal{U}(\mathbb{K}(\mathcal{L}) \otimes B)$.

On the other hand, $B^{\prime}=\left\{\left(\omega \otimes \mathrm{id}_{\mathcal{L}}\right) \mathbb{F} \mid \omega \in \mathbb{B}(\mathcal{L})_{*}\right\}^{\mathrm{CLS}}$ is a $\mathrm{C}^{*}$-algebra. Therefore $B^{\prime} \subseteq \mathcal{M}(B)$ and

$$
\begin{aligned}
B^{\prime} B & =\left\{\left(\omega \otimes \mathrm{id}_{\mathbb{K}(\mathcal{L})}\right) \mathbb{F}\left(1_{\mathcal{L}} \otimes b\right) \mid \omega \in \mathbb{B}(\mathcal{L})_{*}, b \in B\right\}^{\text {-closed linear span }} \\
& =\left\{\left(\omega \otimes \mathrm{id}_{\mathbb{K}(\mathcal{L})}\right) \mathbb{F}(m \otimes b) \mid m \in \mathbb{K}(\mathcal{L}), \omega \in \mathbb{B}(\mathcal{L})_{*}, b \in B\right\}^{\text {-closed linear span }} \\
& =\left\{\left(\omega \otimes \mathrm{id}_{\mathbb{K}(\mathcal{L})}\right)(m \otimes b) \mid m \in \mathbb{K}(\mathcal{L}), \omega \in \mathbb{B}(\mathcal{L})_{*}, b \in B\right\}^{\text {-closed linear span }}=B .
\end{aligned}
$$

Proposition 5.2. The $\mathrm{C}^{*}$-algebra $B^{\prime}$ coincides with $B$.

Proof. It is sufficient to show that the operators $v$ and $v n$ are affiliated with $B^{\prime}$. Because this will imply the faithful representation $\varphi: B \hookrightarrow \mathbb{B}(\mathcal{L})$ in (1.6) is an element of $\operatorname{Mor}\left(B, B^{\prime}\right)$ and hence $B B^{\prime}=B^{\prime}$.

We consider the following family of unitaries $\left\{T^{\prime}(\lambda)\right\}_{\lambda \in \overline{\mathbb{C}}^{|q|}}$ on $\mathcal{U}(\mathcal{L} \otimes \mathcal{L} \otimes \mathcal{L})$ defined by

$$
T^{\prime}(\lambda)=F_{|q|}\left(\lambda n^{-1} v P \otimes P \otimes v n\right) \mathbb{Y}_{13} .
$$

Using (4.3) and (1.6) we compute

$$
\begin{aligned}
\rtimes\left(v n \otimes 1_{\mathcal{L}}\right) \rtimes^{*} e_{i, j} \otimes e_{k, l}=\zeta^{l j} \rtimes\left(v n \otimes 1_{\mathcal{L}}\right) e_{k, l} \otimes e_{i, j} & =\zeta^{l j} q^{k} \backslash e_{k-1, l+1} \otimes e_{i, j} \\
& =(P \otimes v n) e_{i, j} \otimes e_{k, l} .
\end{aligned}
$$

Combining this with Proposition 4.8 we get

$$
\left(\mathbb{F}^{\lambda}\right)_{12}^{*} \mathbb{F}_{23} \mathbb{F}_{12}^{\lambda} \mathbb{F}_{23}^{*}=\succ_{23} \mathbb{F}_{12}^{\lambda}{{ }^{*}}_{23}^{*}=F_{|q|}\left(\lambda n^{-1} v P \otimes P \otimes v n\right) \mathbb{Y}_{13}=T^{\prime}(\lambda) .
$$

Expression at the extreme left of the above chain of equalities belongs to $\mathcal{U}(\mathbb{K}(\mathcal{L}) \otimes$ $\left.\mathbb{K}(\mathcal{L}) \otimes B^{\prime}\right)$, so is $F_{|q|}\left(\lambda n^{-1} v P \otimes P \otimes v n\right) \mathbb{Y}_{13}$ for all $\lambda \in \overline{\mathbb{C}}^{|q|}$. Now $\mathbb{Y} \in \mathcal{U}(\mathcal{L} \otimes \mathcal{L})$ and $X \eta \mathbb{K}(\mathcal{L}) \otimes \mathbb{K}(\mathcal{L})$ with $\operatorname{Spec}(X)=\overline{\mathbb{C}}^{|q|}$. Then, by virtue of 24, Proposition $5.2]$, the map $\overline{\mathbb{C}}^{|q|} \ni \lambda \rightarrow F_{|q|}(\lambda X) \in \mathcal{M}(\mathbb{K}(\mathcal{L}) \otimes \mathbb{K}(\mathcal{L}))$ is strictly continuous and so is the map $\lambda \rightarrow \mathbb{F}^{\lambda}=\mathbb{F}(\lambda X) \mathbb{Y}$. Therefore, $\left\{T^{\prime}(\lambda)\right\}_{\lambda \in \overline{\mathbb{C}}^{|q|}}$ is a strictly continuous family of elements of $\mathcal{U}\left(\mathbb{K}(\mathcal{L}) \otimes \mathbb{K}(\mathcal{L}) \otimes B^{\prime}\right)$. In particular, for $\lambda=0$ we have $T^{\prime}(0)=\mathbb{Y}_{13}$; hence $\mathbb{Y} \in \mathcal{U}\left(\mathbb{K}(\mathcal{L}) \otimes B^{\prime}\right)$. Now the slices $\left(\omega \otimes \operatorname{id}_{\mathbb{K}(\mathcal{L})}\right) \mathbb{Y}$ for $\omega \in \mathbb{B}(\mathcal{L})_{*}$ are dense in $\mathrm{C}(\mathbb{T})$ and $v \in \mathrm{C}(\mathbb{T})$ imply $v \in \mathcal{M}\left(B^{\prime}\right)$. Also the map $\overline{\mathbb{C}}^{|q|} \ni \lambda \rightarrow F_{|q|}\left(\lambda n^{-1} v P \otimes P \otimes v n\right)=T^{\prime}(\lambda) T^{\prime}(0)^{*} \in \mathcal{U}\left(\mathbb{K}(\mathcal{L}) \otimes \mathbb{K}(\mathcal{L}) \otimes B^{\prime}\right)$ is strictly 
continuous. Finally, combining [24, Proposition 5.2] and [19, Proposition 6.11] give $\left(n^{-1} v P \otimes P \otimes v n\right) \eta \mathbb{K}(\mathcal{L}) \otimes \mathbb{K}(\mathcal{L}) \otimes B^{\prime}$ and consequently $v n \eta B^{\prime}$.

5.2. Construction of the comultiplication map. Recall the faithful representation $\varphi: B \rightarrow \mathbb{B}(\mathcal{H})$ in (1.6), the unitary operators $z \in \mathcal{U}(\mathcal{H})$ and $\mathbb{U} \in \mathcal{U}(\mathcal{L} \otimes \mathcal{H})$ from the Section 4. Also recall the actions of $z \in \mathcal{U}(\mathcal{H})$ and $\mathbb{U} \in \mathcal{U}(\mathcal{L} \otimes \mathcal{H})$ on the canonical basis elements $e_{p}$ and $e_{i, j} \otimes e_{p}$ are defined by $z\left(e_{p}\right)=e_{p+1}$ and $\mathbb{U}\left(e_{i, j} \otimes e_{p}\right)=e_{i, j} \otimes e_{p+j}$, respectively. We observe that $\mathbb{U}$ commutes with $v \otimes 1_{\mathcal{H}}$ and

$$
\mathbb{U}\left(n \otimes 1_{\mathcal{H}}\right) \mathbb{U}^{*}\left(e_{i, j} \otimes e_{p}\right)=q^{i} e_{i, j+1} \otimes e_{p+1}=n \otimes z\left(e_{i, j} \otimes e_{p}\right) .
$$

Therefore the map $\delta: B \rightarrow B \otimes \mathrm{C}(\mathbb{T})$ given by

$$
\delta(v):=v \otimes 1_{\mathrm{C}(\mathbb{T})}, \quad \delta(n)=n \otimes z .
$$

is a well defined action of $\mathbb{T}$ on $B$. Consequently, $(B, \delta)$ is an object of $\mathfrak{C}^{*} \mathfrak{a l g}(\mathbb{T})$ and $(\mathbb{U}, \varphi)$ is a faithful covariant representation of $(B, \delta, \mathbb{T})$ on $\mathcal{L}$. Next we define the canonical embeddings $j_{1}, j_{2} \in \operatorname{Mor}\left(B, B \otimes_{\mathrm{R}} B\right)$ in (2.13) using the braiding unitary $\rtimes$ defined in (4.3). On the generators $v$ and $n$

$$
\begin{array}{ll}
j_{1}(v):=v \otimes 1_{\mathcal{L}}, & j_{2}(v):=\rtimes\left(v \otimes 1_{\mathcal{L}}\right){ }^{*}=Z\left(1_{\mathcal{L}} \otimes v\right) Z^{*}=1_{\mathcal{L}} \otimes v, \\
j_{1}(n):=n \otimes 1_{\mathcal{L}}, & j_{2}(n):=\rtimes\left(n \otimes 1_{\mathcal{L}}\right){ }^{*}=Z\left(1_{\mathcal{L}} \otimes n\right) Z^{*}=P \otimes n,
\end{array}
$$

where $P \in \mathcal{U}(\mathcal{L})$ is defined in (4.5). Observe that $j_{1}(n), j_{2}(n) \eta B \otimes_{\mathrm{R}} B$.

Define $\Delta_{B}(b):=\mathbb{F}\left(b \otimes 1_{\mathcal{L}}\right) \mathbb{F}^{*}$ for all $b \in B$. By Theorem [3.6. $\left(B, \Delta_{B}\right)$ is the braided $\mathrm{C}^{*}$-quantum group over $\mathbb{T}$ generated by $\mathbb{F}$.

Let us compute $\Delta_{B}$ on the generators $v, n$. Since $\mathbb{Y}$ generates $\mathbb{T}$ then $\mathbb{Y}(v \otimes$ $\left.1_{\mathcal{L}}\right) \mathbb{Y}^{*}=v \otimes v$. Also $v \otimes v\left(e_{i, j} \otimes e_{k, l}\right)=e_{i-1, j} \otimes e_{k-1, l}$ shows that it commutes with both the operators $|X|$ and $\Phi_{X}$ in (4.4), so with $X$. Therefore,

$$
\Delta_{B}(v):=\mathbb{F}\left(v \otimes 1_{\mathcal{L}}\right) \mathbb{F}^{*}=F_{|q|}(X) \mathbb{Y}\left(v \otimes 1_{\mathcal{L}}\right) \mathbb{Y}^{*} F_{|q|}(X)^{*}=v \otimes v=j_{1}(v) j_{2}(v) .
$$

A simple computation shows that $R:=j_{1}(n) j_{2}\left(v^{*}\right)=n \otimes v^{*}, S:=j_{1}(v) j_{2}(n)=$ $v P \otimes n$, and

$$
X=R^{-1} S=\left(j_{1}(n) j_{2}\left(v^{*}\right)\right)^{-1} j_{1}(v) j_{2}(n)=n^{-1} v P \otimes v n .
$$

Then $R$ and $S$ are unbounded densely defined normal operators and satisfy the commutation relations [24, (0.1)]. Since $X=R^{-1} S$ is also a normal normal operator with spectrum $\overline{\mathbb{C}}^{|q|},[24$, Theorem 2.1-2.2] apply and show that $R+S$ is normal with spectrum $\overline{\mathbb{C}}^{|q|}$ and

$F_{|q|}(X)\left(n \otimes v^{*}\right) F_{|q|}(X)^{*}=F_{|q|}\left(R^{-1} S\right) R F_{|q|}\left(R^{-1} S\right)^{*}=R \dot{+} S=n \otimes v^{*} \dot{+} v P \otimes n$.

The following computation

$$
\mathbb{Y}\left(n \otimes 1_{\mathcal{L}}\right) \mathbb{Y}^{*}\left(e_{i, j} \otimes e_{k, l}\right)=q^{i} e_{i, j+1} \otimes e_{k+1, l}=n \otimes v^{*}\left(e_{i, j} \otimes e_{k, l}\right) .
$$

gives $\Delta_{B}(n):=\mathbb{F}\left(n \otimes 1_{\mathcal{L}}\right) \mathbb{F}^{*}=j_{1}(n) j_{2}\left(v^{*}\right) \dot{+} j_{1}(v) j_{2}(n)$. Now, $n \eta B$ and $\Delta_{B} \in$ $\operatorname{Mor}\left(B, B \bigotimes_{\mathrm{R}} B\right)$ imply $\Delta_{B}(n) \eta B \bigotimes_{\mathrm{R}} B$. Thus $\Delta_{B}$ in (1.5) extends to a coassociative element of $\operatorname{Mor}^{\mathbb{T}}\left(B, B \bigotimes_{\mathrm{R}} B\right)$ and satisfies the cancellation conditions. In summary, we obtain the following result.

Theorem 5.9. The pair $\left(B, \Delta_{B}\right)$ is the braided $\mathrm{C}^{*}$-quantum group over $\mathbb{T}$ generated by the braided multiplicative unitary $\mathbb{F}$.

Remark 5.10. Define $p \in \operatorname{Mor}(B, B)$ by $p(v)=v$ and $p(n)=0$. Existence of $p$ is guaranteed by the universal property of $B$. Clearly, $\operatorname{Im}(p)=\mathrm{C}(\mathbb{T})$. Furthermore, the restriction of the action $\delta$ of $\mathbb{T}$ on $\mathrm{C}(\mathbb{T})$ is trivial and (5.6) implies $\Delta_{B}(v)=$ 
$v \otimes v=\Delta_{\mathrm{C}(\mathbb{T})}(v)$. This shows that $p \in \operatorname{Mor}^{\mathbb{T}}(B, \mathrm{C}(\mathbb{T}))$ and satisfies $\left(p \bigotimes_{\mathrm{R}} p\right) \Delta_{B}(v)=$ $\Delta_{\mathrm{C}(\mathbb{T})}(p(v))$. Hence, $\mathbb{T}$ is a closed quantum subgroup of braided $\mathrm{E}_{\mathrm{q}}(2)$.

If $q \in \mathbb{R}$, then $\zeta=1$ which implies the braiding operator $\lambda=\Sigma$ in (4.3). Consequently, $\mathbb{F}$ is an ordinary manageable multiplicative unitary, $\otimes_{\mathrm{R}}$ coincides with $\otimes$ and (1.5) also coincides with (1.3). In conclusion, we have

Corollary 5.11. For real deformation parameters $0<q<1$, the deformation $\left(B, \Delta_{B}\right)$ coincide with Woronowicz's $\mathrm{E}_{\mathrm{q}}(2)$ groups.

5.3. The bosonisation. The $R$-matrix in (1.4) corresponds to the group homomorphism $\mathbb{Z} \ni m \rightarrow \mathrm{R}(\cdot, m) \in \widehat{\mathbb{Z}} \cong \mathbb{T}$ and it induces unique representation $\mathbb{V} \in$ $\mathcal{U}\left(\mathbb{K}(\mathcal{L}) \otimes \mathrm{C}_{0}(\mathbb{Z})\right) \subset \mathcal{U}(\mathcal{L} \otimes \mathcal{H})$ of $\mathbb{Z}$ on $\mathcal{L}$ defined by $\mathbb{V}\left(e_{i, j} \otimes e_{p}\right)=\zeta^{-p j} e_{i, j} \otimes e_{p}$ satisfying (A.3). Define $\hat{\mathbb{V}} \in \mathcal{U}\left(\mathrm{C}_{0}(\mathbb{Z}) \otimes \mathbb{K}(\mathcal{L})\right) \subset \mathcal{U}(\mathcal{H} \otimes \mathcal{L})$ by

$$
\widehat{\mathbb{V}} e_{p} \otimes e_{i, j}=\zeta^{p j} e_{p} \otimes e_{i, j}
$$

By virtue of Theorem 3.6 the managable braided multiplicative unitary $\mathbb{F} \in \mathcal{U}(\mathcal{L} \otimes \mathcal{L})$ constructed in Theorem 4.6 over $\mathbb{T}$ relative to $(\mathrm{U}, \mathrm{R})$ is also a manageable braided multiplicative unitary over $\mathbb{T}$ in the sense of [15, Definition 3.2].

According to [15, Theorem $3.7 \& 3.8$ ]

$$
\mathcal{W}:=\mathbb{W}_{13} \mathbb{U}_{23} \hat{\mathbb{V}}_{34}^{*} \mathbb{F}_{24} \hat{\mathbb{V}}_{34} \quad \text { in } \mathcal{U}(\mathcal{H} \otimes \mathcal{L} \otimes \mathcal{H} \otimes \mathcal{L})
$$

is a manageable multiplicative unitary, where $\mathbb{U}$ is the concrete realisation of $\mathrm{U}$ on $\mathcal{L} \otimes \mathcal{H}$. Let $\mathcal{W}$ generates the $\mathrm{C}^{*}$-quantum group $\mathbb{H}=\left(C, \Delta_{C}\right)$.

The $\mathrm{C}^{*}$-algebra $C:=\mathrm{C}(\mathbb{T}) \otimes_{\mathrm{R}} B=j_{\mathrm{C}(\mathbb{T})}(\mathrm{C}(\mathbb{T})) j_{B}(B) \subset \mathbb{B}(\mathcal{H} \otimes \mathcal{L})$ where, $j_{\mathrm{C}(\mathbb{T})}(z)=z \otimes 1_{\mathcal{L}}$ and $j_{B}(b)=\hat{\mathbb{V}}^{*}\left(1_{\mathcal{H}} \otimes b\right) \hat{\mathbb{V}}$, where $z$ is the unitary generator of $\mathrm{C}(\mathbb{T})$ and $b \in B \subset \mathbb{B}(\mathcal{L})$. The concrete realisations of $v, n, z$ give

$$
\begin{array}{ll}
j_{B}(v)=1_{\mathcal{H}} \otimes v, & j_{\mathrm{C}(\mathbb{T})}(z) j_{B}(v)=j_{B}(v) j_{\mathrm{C}(\mathbb{T})}(z), \\
j_{B}(n)=P^{\prime} \otimes n, & j_{\mathrm{C}(\mathbb{T})}(z) j_{B}(n)=\zeta j_{B}(n) j_{\mathrm{C}(\mathbb{T})}(z),
\end{array}
$$

where $P^{\prime} \in \mathcal{U}(\mathcal{H})$ defined by $P^{\prime} e_{p}=\zeta^{-p} e_{p}$. Equivalently, $C=B \rtimes \mathbb{Z}$ with respect to the action of $\mathbb{Z}$ on $B$ given by $(k, v) \rightarrow v$ and $(k, n) \rightarrow \zeta^{k} n$ for all $k \in \mathbb{Z}$.

Recall the identification $\mathcal{L} \cong \mathcal{H} \otimes \mathcal{H}$. We have already observed $\mathbb{U}=\mathbb{W}_{23} \in$ $\mathcal{U}\left(\mathcal{H}^{\otimes 3}\right)$. Using these we rewrite (5.13) as

$$
\mathcal{W}=\mathbb{W}_{14} \mathbb{W}_{34} \hat{\mathbb{V}}_{456}^{*} F_{|q|}\left(n^{-1} v P \otimes v n\right)_{2356} \mathbb{W}_{25} \mathbb{W}_{35} \hat{\mathbb{V}}_{456} \quad \text { in } \mathcal{U}\left(\mathcal{H}^{\otimes 6}\right) .
$$

Since $\hat{\mathbb{V}}_{456}$ acts trivially on the fifth leg it commutes with $\mathbb{W}_{25} \mathbb{W}_{35}$. Moreover,

$$
\hat{\mathbb{V}}^{*}\left(1_{\mathcal{H}} \otimes v n\right) \hat{\mathbb{V}}=j_{B}(v n)=P^{\prime} \otimes v n .
$$

Thus, (5.14) gets simplified as

$$
\mathcal{W}=\mathbb{W}_{14} \mathbb{W}_{34} F_{|q|}\left(n^{-1} v P \otimes P^{\prime} \otimes v n\right)_{23456} \mathbb{W}_{25} \mathbb{W}_{35} \quad \text { in } \mathcal{U}\left(\mathcal{H}^{\otimes 6}\right) .
$$

and the comultiplication map $\Delta_{C} \in \operatorname{Mor}(C, C \otimes C)$ is given by $\Delta_{C}(c)=\mathcal{W}(c \otimes$ $\left.1_{\mathcal{H} \otimes^{\otimes 3}}\right) \mathcal{W}^{*}$ for all $c \in C$. Clearly,

$$
\Delta_{C}\left(j_{\mathrm{C}(\mathbb{T})}(z)\right)=j_{\mathrm{C}(\mathbb{T})}(z) \otimes j_{\mathrm{C}(\mathbb{T})}(z), \quad \Delta_{C}\left(j_{B}(v)\right)=j_{B}(v) \otimes j_{B}(v) .
$$

Next we compute $\Delta_{C}\left(j_{B}(n)\right)$. By (5.8) we have

$$
\mathbb{W}_{25} \mathbb{W}_{35}\left(j_{B}(n) \otimes 1_{\mathcal{H} \otimes 3}\right) \mathbb{W}_{35}^{*} \mathbb{W}_{25}^{*}=P^{\prime} \otimes n \otimes 1_{\mathcal{H}} \otimes v^{*},
$$

Furthermore, a variant of (5.7) gives

$$
\begin{aligned}
& F_{|q|}\left(n^{-1} v P \otimes P^{\prime} \otimes v n\right)_{23456}\left(P^{\prime} \otimes n \otimes 1_{\mathcal{H}} \otimes v^{*}\right) F_{|q|}\left(n^{-1} v P \otimes P^{\prime} \otimes v n\right)_{23456}^{*} \\
& =P^{\prime} \otimes n \otimes 1_{\mathcal{H}} \otimes v^{*} \dot{+} P^{\prime} \otimes v P \otimes P^{\prime} \otimes n .
\end{aligned}
$$


Now using the concrete realisation of the operators $v, n, P, P^{\prime}, \mathbb{W}$ we compute

$$
\begin{aligned}
\mathbb{W}_{14} \mathbb{W}_{34}\left(P^{\prime} \otimes n \otimes 1_{\mathcal{H}}\right) e_{p} \otimes e_{i, j} \otimes e_{s} & =\zeta^{-p} q^{i} e_{p} \otimes e_{i, j+1} \otimes e_{s+j+1+p} \\
& =\left(\left(P^{\prime} \otimes n \otimes z\right) \mathbb{W}_{14} \mathbb{W}_{34}\right) e_{p} \otimes e_{i, j} \otimes e_{s},
\end{aligned}
$$

and

$$
\begin{aligned}
\mathbb{W}_{14} \mathbb{W}_{34}\left(P^{\prime} \otimes v P \otimes P^{\prime}\right) e_{p} \otimes e_{i, j} \otimes e_{q} & =\zeta^{-p-s-j} e_{p} \otimes e_{i-1, j} \otimes e_{s+p+j} \\
& =\left(\left(1_{\mathcal{H}} \otimes v \otimes P^{\prime}\right) \mathbb{W}_{14} \mathbb{W}_{34}\right) e_{p} \otimes e_{i, j} \otimes e_{s}
\end{aligned}
$$

Combining the last four calculations we obtain

$$
\Delta_{C}\left(j_{B}(n)\right)=j_{B}(n) \otimes j_{\mathrm{C}(\mathbb{T})}(z) j_{B}\left(v^{*}\right) \dot{+} j_{B}(v) \otimes j_{B}(n) .
$$

Summarising, we have the bosonisation of $\mathrm{E}_{\mathrm{q}}(2)$.

Theorem 5.15. Let $C$ be the universal $\mathrm{C}^{*}$-algebra generated by the the unitaries $\tilde{z}, \tilde{v}$ and the normal operator $\tilde{n}$ with $\operatorname{Sp}(\tilde{n})=\overline{\mathbb{C}}^{|q|}$ subject to the commutation relations

$$
\tilde{z} \tilde{v} \tilde{z}^{*}=\tilde{v}, \quad \tilde{z} \tilde{n} \tilde{z}^{*}=\zeta \tilde{n}, \quad \tilde{v} \tilde{n} \tilde{v}^{*}=q \tilde{n} .
$$

There exists a unique $\Delta_{C} \in \operatorname{Mor}(C, C \otimes C)$ such that

$$
\Delta_{C}(\tilde{z})=\tilde{z} \otimes \tilde{z}, \quad \Delta_{C}(\tilde{v})=\tilde{v} \otimes \tilde{v}, \quad \Delta_{C}(\tilde{n})=\tilde{n} \otimes \tilde{z} \tilde{v}^{*} \dot{+} \tilde{v} \otimes \tilde{n}
$$

and $\mathbb{H}=\left(C, \Delta_{C}\right)$ is a $\mathrm{C}^{*}$-quantum group. Moreover, there exists an idempotent Hopf ${ }^{*}$-homomorphism $f \in \operatorname{Mor}(C, C)$ with $f(\tilde{z})=\tilde{z}, f(\tilde{v})=1_{C}$ and $f(\tilde{n})=0$. Its image is the copy of $\mathrm{C}(\mathbb{T})$ generated by $\tilde{z}$ as a closed quantum subgroup of $\mathbb{H}$ and its kernel is the copy of $B$ generated by $\tilde{v}, \tilde{n}$ as the braided $\mathrm{E}_{\mathrm{q}}(2)$ group over $\mathbb{T}$.

6. Contraction procedure Between braided $\mathrm{SU}_{q}(2)$ and $\mathrm{E}_{q}(2)$ Groups AND THEIR RESPECTIVE BOSONISATIONS

Throughout this section we fix a complex deformation parameter $0<|q|<1$, the unitary $R$-matrix $\mathrm{R}: \mathbb{Z} \times \mathbb{Z} \rightarrow \mathbb{T}$, defined by (1.4) and $\mathbb{T}$ is a quasitriangular compact quantum group with respect to $\mathrm{R}$. We denote the braided $\mathrm{E}_{\mathrm{q}}(2)$ constructed in Theorem 5.9 by $\left(B_{\mathrm{E}_{\mathrm{q}}(2)}, \Delta_{\mathrm{E}_{\mathrm{q}}(2)}\right)$.

Denote $\mathbb{N}^{0}=\mathbb{N} \cup\{0\}$. Consider the Hilbert space $\mathcal{L}_{\mathrm{SU}_{\mathrm{q}}(2)}=\ell^{2}\left(\mathbb{N}^{0} \times \mathbb{Z}\right)$ equipped

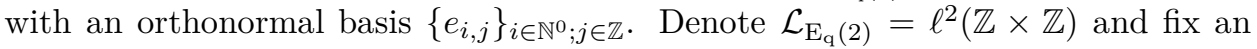
orthonormal basis $\left\{e_{i, j}\right\}_{i, j \in \mathbb{Z}}$ for it. Recall that $\mathcal{L}_{\mathrm{E}_{\mathrm{q}}(2)}$ is an object of $\mathfrak{R e p}(\mathbb{T})$ with respect to the representation $\mathbb{U}\left(e_{i, j} \otimes e_{p}\right)=e_{i, j} \otimes e_{p+j}$ defined in Section 4 Identification of the basis vectors of $\mathcal{L}_{\mathrm{SU}_{\mathrm{q}}(2)}$ with the corresponding basis vectors of $\mathcal{L}_{\mathrm{E}_{\mathrm{q}}(2)}$ defines an embedding $\mathcal{L}_{\mathrm{SU}_{\mathrm{q}}(2)} \hookrightarrow \mathcal{L}_{\mathrm{E}_{\mathrm{q}}(2)}$. Furthermore, the restriction of $\mathbb{U}$ on $\mathcal{L}_{\mathrm{SU}_{\mathrm{q}}(2)}$ defines a representation of $\mathbb{T}$ on it. Consequently, we obtain a $\mathbb{T}$-equivariant embedding $\mathbb{B}\left(\mathcal{L}_{\mathrm{SU}_{\mathrm{q}}(2)}\right) \hookrightarrow \mathbb{B}\left(\mathcal{L}_{\mathrm{E}_{\mathrm{q}}(2)}\right)$ and $1_{\mathcal{L}_{\mathrm{SU}_{\mathrm{q}}(2)}}$ is a $\mathbb{T}$-equivariant orthogonal projection onto $\mathcal{L}_{\mathrm{SU}_{\mathrm{q}}(2)}$.

Now we recall the braided $\mathrm{SU}_{\mathrm{q}}(2)=\left(B_{\mathrm{SU}_{\mathrm{q}}(2)}, \Delta_{\mathrm{SU}_{\mathrm{q}}(2)}\right)$ group over $\mathbb{T}$ with respect to $\mathrm{R}$, constructed in [8]. Define $\alpha, \gamma \in \mathbb{B}\left(\mathcal{L}_{\mathrm{SU}_{\mathrm{q}}(2)}\right)$ by

$$
\alpha e_{i, j}:=\sqrt{1-|q|^{2 i}} e_{i-1, j}, \quad \gamma e_{i, j}:=q^{i} e_{i, j-1} .
$$

Then $B_{\mathrm{SU}_{\mathrm{q}}(2)}$ is the universal $\mathrm{C}^{*}$-algebra generated by $\alpha$ and $\gamma$. Define $f_{\alpha}, f_{\gamma} \in$ $\mathrm{C}_{0}\left(\overline{\mathbb{C}}^{|q|}\right)$ by $f_{\alpha}(\lambda)=\sqrt{1-|\lambda|^{2}} \chi(\lambda)$ and $f_{\gamma}(\lambda)=\bar{\lambda} \chi(\lambda)$, where $\chi$ is the indicator function of the closed unit disc $\{z \in \mathbb{C}|| z \mid \leq 1\}$. By the property of the continuous functional calculus we have

$$
\alpha=v f_{\alpha}(n), \quad \gamma=f_{\gamma}(n) .
$$

Following similar arguments as in [25, Section 1] and replacing $\mu$ by $|q|$ it is easy to observe

$$
B_{\mathrm{SU}_{\mathrm{q}}(2)} \subset B_{\mathrm{E}_{\mathrm{q}}(2)} \quad \text { and } \quad B_{\mathrm{SU}_{\mathrm{q}}(2)}=1_{\mathcal{L}_{\mathrm{SU}_{\mathrm{q}}(2)}} B_{\mathrm{E}_{\mathrm{q}}(2)} 1_{\mathcal{L}_{\mathrm{SU}_{\mathrm{q}}(2)}} .
$$


Then the restriction of $\delta$ in (5.5) $\delta: B_{\mathrm{SU}_{\mathrm{q}}(2)} \rightarrow B_{\mathrm{SU}_{\mathrm{q}}(2)} \otimes \mathrm{C}(\mathbb{T})$ defines an action of $\mathbb{G}$ on $B_{\mathrm{SU}_{\mathrm{q}}(2)}$. It is easy to verify that $\delta(\alpha)=\alpha \otimes 1_{\mathrm{C}(\mathbb{T})}$ and $\delta(\gamma)=\gamma \otimes z^{*}$ is an action of $\mathbb{T}$ on $B_{\mathrm{SU}_{\mathrm{q}}(2)}$, where $z$ is the unitary generator of $\mathrm{C}(\mathbb{T})$ defined in the beginning of Section 4 . In fact, $\delta(b)=\mathbb{U}\left(b \otimes 1_{\mathrm{C}(\mathbb{T})}\right) \mathbb{U}^{*}$ for all $b \in B_{\mathrm{SU}_{\mathrm{q}}(2)}$.

Consequently $B_{\mathrm{SU}_{\mathrm{q}}(2)} \subset B_{\mathrm{E}_{\mathrm{q}}(2)}$ is T-equivariant, $B_{\mathrm{SU}_{\mathrm{q}}(2)} \bigotimes_{\mathrm{R}} B_{\mathrm{SU}_{\mathrm{q}}(2)} \subset B_{\mathrm{E}_{\mathrm{q}}(2)} \bigotimes_{\mathrm{R}}$ $B_{\mathrm{E}_{\mathrm{q}}(2)}$ and the embeddings of $B_{\mathrm{SU}_{\mathrm{q}}(2)}$ into $B_{\mathrm{SU}_{\mathrm{q}}(2)} \otimes_{\mathrm{R}} B_{\mathrm{SU}_{\mathrm{q}}(2)}$ are obtained by restricting $j_{1}, j_{2} \in \operatorname{Mor}\left(B_{\mathrm{E}_{\mathrm{q}}(2)}, B_{\mathrm{E}_{\mathrm{q}}(2)} \bigotimes_{\mathrm{R}} B_{\mathrm{E}_{\mathrm{q}}(2)}\right)$ in (5.6). In fact, a simple computation using (6.2) and (4.5) give $j_{1}(\alpha)=\alpha \otimes 1_{\mathcal{L}_{\mathrm{E}_{\mathrm{q}}(2)},} j_{2}(\alpha)=1_{\mathcal{L}_{\mathrm{E}_{\mathrm{q}}(2)}} \otimes \alpha$, $j_{1}(\gamma)=\gamma \otimes 1_{\mathcal{L}_{\mathrm{E}_{\mathrm{q}}(2)}}$ and $j_{2}(\gamma)=P^{*} \otimes \gamma$, where $P \in \mathcal{U}\left(\mathcal{L}_{\mathrm{E}_{\mathrm{q}}(2)}\right)$ defined in (4.5).

The comultiplication map $\Delta_{\mathrm{SU}_{\mathrm{q}}(2)}: B_{\mathrm{SU}_{\mathrm{q}}(2)} \rightarrow B_{\mathrm{SU}_{\mathrm{q}}(2)} \bigotimes_{\mathrm{R}} B_{\mathrm{SU}_{\mathrm{q}}(2)}$ is defined by

$$
\begin{aligned}
& \Delta_{\mathrm{SU}_{\mathrm{q}}(2)}(\alpha)=j_{1}(\alpha) j_{2}(\alpha)-q j_{1}(\gamma)^{*} j_{2}(\gamma), \\
& \Delta_{\mathrm{SU}_{\mathrm{q}}(2)}(\gamma)=j_{1}(\gamma) j_{2}(\alpha)+j_{1}(\alpha)^{*} j_{2}(\gamma) .
\end{aligned}
$$

Consider the family of inner automorphisms $\left\{\tau^{k}\right\}_{k \in \mathbb{Z}}$ of $B_{\mathrm{E}_{\mathrm{q}}(2)}$ define by $\tau^{k}(a)=$ $v^{k} a v^{-k}$ for all $k \in \mathbb{Z}$. By virtue of (1.1) we have

$$
\tau^{k}(v)=v, \quad \tau^{k}(n)=q^{k} n
$$

Therefore, $\tau^{k}$ is $\mathbb{T}$-equivariant, that is, $\left(\tau^{k} \otimes \mathrm{id}_{\mathrm{C}(\mathbb{T})}\right) \circ \delta=\delta \circ \tau^{k}$ for all $k \in \mathbb{Z}$. Similarly, using (1.5) we observe that $\tau^{k}$ is an automorphism of braided $\mathrm{C}^{*}$-quantum groups:

$$
\left(\tau^{k} \bigotimes_{\mathrm{R}} \tau^{k}\right) \circ \Delta_{\mathrm{E}_{\mathrm{q}}(2)}=\Delta_{\mathrm{E}_{\mathrm{q}}(2)} \circ \tau^{k} \quad \text { for all } k \in \mathbb{Z} .
$$

The braided analogue of the contraction procedure [25] is contained is following result.

Theorem 6.6. For any $a \in B_{\mathrm{E}_{\mathrm{q}}(2)}$

$$
\Delta_{\mathrm{E}_{\mathrm{q}}(2)}(a)=\lim _{k \rightarrow \infty}\left(\tau^{k} \otimes_{\mathrm{R}} \tau^{k}\right) \Delta_{\mathrm{SU}_{\mathrm{q}}(2)}\left(1_{\mathcal{L}_{\mathrm{SU}_{\mathrm{q}}(2)}}\left(\tau^{-k}(a)\right) 1_{\mathcal{L}_{\mathrm{SU}_{\mathrm{q}}(2)}}\right) .
$$

Proof. The proof essentially follows from [25, Sections $2 \& 3$ ] replacing $\mu$ by $|q|$, tensor product $\otimes$ of $\mathrm{C}^{*}$-algebras by $\otimes_{\mathrm{R}}$ and taking care of certain commutation relations.

Recall the dense ${ }^{*}$-subalgebra $\mathcal{B}_{\mathrm{E}_{\mathrm{q}}(2)}$ of $B_{\mathrm{E}_{\mathrm{q}}(2)}$ defined in [25, Equation 22]

$$
\mathcal{B}_{\mathrm{E}_{\mathrm{q}}(2)}=\cup_{k \in \mathbb{Z}} \tau^{l}\left(B_{\mathrm{SU}_{\mathrm{q}}(2)}\right) .
$$

Suppose $a \in \mathcal{B}_{\mathrm{E}_{\mathrm{q}}(2)}$. For sufficiently large $k, \tau^{-k}(a) \in B_{\mathrm{SU}_{\mathrm{q}}(2)}$, which implies, $\Delta_{\mathrm{SU}_{\mathrm{q}}(2)}\left(\tau^{-k}(a)\right) \in B_{\mathrm{SU}_{\mathrm{q}}(2)} \bigotimes_{\mathrm{R}} B_{\mathrm{SU}_{\mathrm{q}}(2)}$. Also, $a$ commutes with $1_{\mathcal{L}_{\mathrm{SU}_{\mathrm{q}}(2)}}$. Therefore, (6.7) coincides with the following expression

$$
\Delta_{\mathrm{E}_{\mathrm{q}}(2)}(a)=\lim _{k \rightarrow \infty}\left(\tau^{k} \bigotimes_{\mathrm{R}} \tau^{k}\right) \Delta_{\mathrm{SU}_{\mathrm{q}}(2)}\left(\tau^{-k}(a)\right) \quad \text { for all } a \in \mathcal{B}_{\mathrm{E}_{\mathrm{q}}(2)} .
$$

Define the following elements

$$
\begin{aligned}
t & =\prod_{k=1}^{\infty}\left(1_{\mathcal{L}_{\mathrm{SU}_{\mathrm{q}}(2)}}-|q|^{2 k} \gamma^{*} \gamma\right) \in B_{\mathrm{SU}_{\mathrm{q}}(2)}, \\
Y & =\sum_{r=0}^{\infty}\left(\prod_{i=1}^{r} \frac{1}{1-|q|^{2 i}}\right)\left(-q j_{1}\left(\gamma^{*}\right) j_{2}(\gamma)\right)^{r}\left(j_{1}(v) j_{2}(v)\right)^{-r} \in B_{\mathrm{E}_{\mathrm{q}}(2)} \bigotimes_{\mathrm{R}} B_{\mathrm{E}_{\mathrm{q}}(2)} .
\end{aligned}
$$

Note that the first term corresponding to $r=0$ is $1_{\mathrm{SU}_{\mathrm{q}}(2)} \bigotimes_{\mathrm{R}} 1_{\mathrm{SU}_{\mathrm{q}}(2)}$. Clearly, $\delta\left(\gamma^{*} \gamma\right)=\gamma^{*} \gamma \otimes 1$. Equivalently, $\gamma^{*} \gamma$ is a degree zero $\mathbb{T}$-homogeneous element of $B_{\mathrm{SU}_{\mathrm{q}}(2)}$. Similarly, each term in $Y$ is also degree zero $\mathbb{T}$-homogeneous element with respect to the diagonal action $\delta \bowtie \delta$ of $\mathbb{T}$ on $B_{\mathrm{E}_{\mathrm{q}}(2)} \bigotimes_{\mathrm{R}} B_{\mathrm{E}_{\mathrm{q}}(2)}$ mentioned in the Section 2.2. Therefore, both the elements $t$ and $Y$ are degree zero $\mathbb{T}$-homogeneous elements. We also observe that $j_{k}(\alpha) j_{l}(\gamma)=j_{l}(\gamma) j_{k}(\alpha)$ for all $k, l=1,2$. 
These give the braided analogue of the formulae [25, Equation 42-43]:

$$
\lim _{k \rightarrow \infty} \alpha^{k} v^{-k}=t^{\frac{1}{2}}, \quad \lim _{k \rightarrow \infty} \Delta_{\mathrm{SU}_{\mathrm{q}}(2)}\left(\alpha^{k}\right)\left(j_{1}(v) j_{2}(v)\right)^{-k}=j_{1}\left(t^{\frac{1}{2}}\right) j_{2}\left(t^{\frac{1}{2}}\right) Y .
$$

Consequently, for $a \in B_{\mathrm{SU}_{\mathrm{q}}(2)}$ we prove that the right hand side of (6.8) is well defined

$$
\begin{aligned}
& \lim _{k \rightarrow \infty}\left[\left(j_{1}(v) j_{2}(v)\right)^{k} \Delta_{\mathrm{SU}_{\mathrm{q}}(2)}\left(v^{-k} t^{\frac{1}{2}}\left(t^{-\frac{1}{2}} a t^{-\frac{1}{2}}\right) t^{\frac{1}{2}} v^{k}\right)\left(j_{1}(v) j_{2}(v)\right)^{-k}\right] \\
& =\lim _{k \rightarrow \infty}\left[\left(j_{1}(v) j_{2}(v)\right)^{k} \Delta_{\mathrm{SU}_{\mathrm{q}}(2)}\left(\left(\alpha^{*}\right)^{k}\left(t^{-\frac{1}{2}} a t^{-\frac{1}{2}}\right) \alpha^{k}\right)\left(j_{1}(v) j_{2}(v)\right)^{-k}\right] \\
& =\widetilde{Y}^{*} \Delta_{\mathrm{SU}_{\mathrm{q}}(2)}(a) \tilde{Y}, \quad \text { where } \tilde{Y}:=\Delta_{\mathrm{SU}_{\mathrm{q}}(2)}\left(t^{-\frac{1}{2}}\right)\left(j_{1}\left(t^{\frac{1}{2}}\right) j_{2}\left(t^{\frac{1}{2}}\right)\right) Y \in B_{\mathrm{E}_{\mathrm{q}}(2)} \otimes_{\mathrm{R}} B_{\mathrm{E}_{\mathrm{q}}(2)} .
\end{aligned}
$$

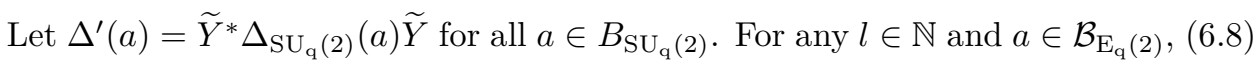
implies $\left(\tau^{l} \bigotimes_{\mathrm{R}} \tau^{l}\right) \Delta^{\prime}(a)=\Delta^{\prime}\left(\tau^{l}(a)\right)$. Also, $\tau^{l} f(n) \rightarrow f(0) 1_{\left.\mathcal{L}_{\mathrm{E}_{\mathrm{q}}(2)}\right)}$ almost uniformly, abbreviated as a.u., as $l \rightarrow \infty$ for all $f \in \mathrm{C}_{0}\left(\overline{\mathbb{C}}^{|q|}\right)$. In particular, we have

$$
\text { a.u. } \lim _{l \rightarrow \infty} \tau^{l}(\alpha)=v, \quad \text { a.u. } \lim _{l \rightarrow \infty} \tau^{l}(\gamma)=0,
$$

Using the second limit in (6.10) we obtain

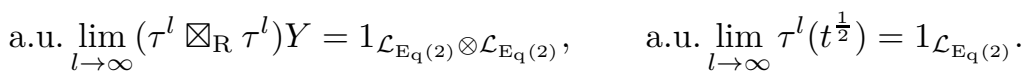

Consequently, the bounded sequence $\left\{a_{l}:=\tau^{l}(t)\right\}_{l \in \mathbb{N}}$ in $B_{\mathrm{E}_{\mathrm{q}}(2)}$ satisfies [25, Equation (39)]

$$
\text { a.u. } \lim _{l \rightarrow \infty} \Delta^{\prime}\left(\tau^{l}(t)\right)=\text { a.u. } \lim _{l \rightarrow \infty}\left(\tau^{l} \bigotimes_{\mathrm{R}} \tau^{l}\right)\left(Y^{*}(t \otimes t) Y\right)=1_{\mathcal{L}_{\mathrm{E}_{\mathrm{q}}(2)} \otimes \mathcal{L}_{\mathrm{E}_{\mathrm{q}}(2)}} \text {. }
$$

Thus, $\Delta^{\prime}$ uniquely extends to an element in $\operatorname{Mor}^{\mathbb{T}}\left(B_{\mathrm{E}_{\mathrm{q}}(2)}, B_{\mathrm{E}_{\mathrm{q}}(2)} \bigotimes_{\mathrm{R}} B_{\mathrm{E}_{\mathrm{q}}(2)}\right)$.

To complete the proof of (6.8), it is sufficient to verify

$$
\lim _{l \rightarrow \infty} \Delta^{\prime}\left(\tau^{l}\left(t^{\frac{1}{2}} \alpha t^{\frac{1}{2}}\right)\right)=\Delta_{\mathrm{E}_{\mathrm{q}}(2)}(v), \quad \lim _{l \rightarrow \infty} \Delta^{\prime}\left(\tau^{l}\left(t^{\frac{1}{2}} \gamma^{*} t^{\frac{1}{2}}\right)\right)=\Delta_{\mathrm{E}_{\mathrm{q}}(2)}(n) .
$$

From the concrete realisation of $n$ and $P$ given by (1.6) and (4.5), it is easy to verify that $n P$ is a normal operator and $\operatorname{Sp}(n P)=\overline{\mathbb{C}}^{|q|}$. Furthermore, (5.6) immediately implies $j_{1}(n) j_{2}(n)=n P \otimes n$. As immediate consequence of [25, Proposition 1.1] we obtain

$$
\lim _{l \rightarrow \infty}|q|^{-l}\left\|\left(\tau^{l}\left(\gamma^{*}\right) \bigotimes_{\mathrm{R}} \tau^{l}(\gamma)\right) \psi\right\|=0, \quad \text { for all } \psi \in \mathcal{D}\left(j_{1}(n)\right) \cap \mathcal{D}\left(j_{2}(n)\right) .
$$

This implies

$$
\lim _{l \rightarrow \infty}|q|^{-l}\left\|\left(\tau^{l} \otimes_{\mathrm{R}} \tau^{l}\right) Y \psi-\psi\right\|=0, \quad \text { for all } \psi \in \mathcal{D}\left(j_{1}(n)\right) \cap \mathcal{D}\left(j_{2}(n)\right) .
$$

Then the proof of (6.12) follows similarly from [25, Section 3 (Step 3)] using (6.4), (6.9) -6.13 .

We end this section with an application of the contraction procedure described above to show that the bosonisations of $\mathrm{SU}_{\mathrm{q}}(2)$ and $\mathrm{E}_{\mathrm{q}}(2)$ are also related via a contraction procedure for the respective braided quantum groups.

Recall the bosonisation $\mathbb{H}=\left(C, \Delta_{C}\right)$ of $\mathrm{E}_{\mathrm{q}}(2)$ described in Theorem 5.15. For any $l \in \mathbb{Z}$ set $\tilde{\tau}^{l}:=1_{\mathrm{C}(\mathbb{T})} \bigotimes_{\mathrm{R}} \tau^{l}$. Then $\left\{\tilde{\tau}^{l}\right\}_{l \in \mathbb{Z}}$ is also a one parameter group of automorphisms of $C=\mathrm{C}(\mathbb{T}) \bigotimes_{\mathrm{R}} B_{\mathrm{E}_{\mathrm{q}}(2)}$. Furthermore, $\tilde{\tau}^{-k}(c) \in B_{\mathrm{U}_{\mathrm{q}}(2)}$ for $c \in C$ and sufficiently large $k$.

Bosonisation of $\mathrm{SU}_{\mathrm{q}}(2)$ is the compact quantum group $\mathrm{U}_{\mathrm{q}}(2)=\left(C_{\mathrm{U}_{\mathrm{q}}(2)}, \Delta_{\mathrm{U}_{\mathrm{q}}(2)}\right)$ with $\mathbb{T}$ as a closed quantum subgroup [8, Section 6]. The underlying $\mathrm{C}^{*}$-algebra $C_{\mathrm{U}_{\mathrm{q}}(2)}$ is the twisted tensor product $\mathrm{C}(\mathbb{T}) \otimes_{\mathrm{R}} B_{\mathrm{SU}_{\mathrm{q}}(2)} \subset \mathbb{B}\left(\mathcal{H} \otimes \mathcal{L}_{\mathrm{SU}_{\mathrm{q}}(2)}\right) \subset \mathbb{B}\left(\mathcal{H} \otimes \mathcal{L}_{\mathrm{E}_{\mathrm{q}}(2)}\right)$. The canonical embeddings $j_{\mathrm{C}(\mathbb{T})}, j_{B_{\mathrm{SU}_{\mathrm{q}}(2)}}$ are give by $j_{\mathrm{C}(\mathbb{T})}(z)=z \otimes 1_{\mathcal{L}_{\mathrm{E}_{\mathrm{q}}(2)}}$ and 
$j_{B_{\mathrm{SU}_{\mathrm{q}}(2)}}(a)=\hat{\mathbb{V}}^{*}\left(1_{\mathcal{H}} \otimes a\right) \hat{\mathbb{V}}$ for $a \in B_{\mathrm{SU}_{\mathrm{q}}(2)} \subset \mathbb{B}\left(\mathcal{L}_{\mathrm{SU}_{\mathrm{q}}(2)}\right)$, where $\hat{\mathbb{V}}$ is the unitary in (5.12). Consequently, (6.3) implies

$$
C_{\mathrm{U}_{\mathrm{q}}(2)} \subset C \quad \text { and } \quad C_{\mathrm{U}_{\mathrm{q}}(2)}=1_{\mathcal{H} \otimes \mathcal{L}_{\mathrm{SU}_{\mathrm{q}}(2)}} C 1_{\mathcal{H} \otimes \mathcal{L}_{\mathrm{SU}_{\mathrm{q}}(2)}}
$$

From [14, Theorem 6.4], the comultiplication $\Delta_{\mathrm{U}_{\mathrm{q}}(2)}$ is given by $\Delta_{\mathrm{U}_{\mathrm{q}}(2)}:=\Psi \circ$ $\left(\operatorname{id}_{\mathrm{C}(\mathbb{T})} \otimes_{\mathrm{R}} \Delta_{\mathrm{SU}_{\mathrm{q}}(2)}\right)$, where

$$
\Psi(x):=\mathbb{W}_{13} \mathbb{U}_{23} \hat{\mathbb{V}}_{34}^{*} x_{124} \hat{\mathbb{V}}_{34} \mathbb{U}_{23}^{*} \mathbb{W}_{13} \quad \text { for } x \in \mathbb{B}\left(\mathcal{H} \otimes \mathcal{L}_{\mathrm{E}_{\mathrm{q}}(2)} \otimes \mathcal{L}_{\mathrm{E}_{\mathrm{q}}(2)}\right) .
$$

We also observe that $\Delta_{C}=\Psi \circ\left(\operatorname{id}_{\mathrm{C}(\mathbb{T})} \otimes_{\mathrm{R}} \Delta_{\mathrm{E}_{\mathrm{q}}(2)}\right)$. In particular, we get $\left.\Delta_{C}\right|_{\mathrm{C}(\mathbb{T})}=$ $\left.\Delta_{\mathrm{U}_{\mathrm{q}}(2)}\right|_{\mathrm{C}(\mathbb{T})}=\Delta_{\mathrm{C}(\mathbb{T})}$ as expected.

Corollary 6.14. Contraction of $\mathrm{U}_{\mathrm{q}}(2)$ group is isomorphic to the bosonisation of braided $\mathrm{E}_{\mathrm{q}}(2)$ group. Equivalently,

$$
\Delta_{C}(c)=\lim _{k \rightarrow \infty}\left(\tilde{\tau}^{k} \otimes \tilde{\tau}^{k}\right) \Delta_{\mathrm{U}_{\mathrm{q}}(2)}\left(1_{\mathcal{H} \otimes \mathcal{L}_{\mathrm{E}_{\mathrm{q}}(2)}}\left(\tilde{\tau}^{-k}(c)\right) 1_{\mathcal{H} \otimes \mathcal{L}_{\mathrm{E}_{\mathrm{q}}(2)}}\right) \quad \text { for all } c \in C .
$$

Proof. Since $\mathcal{B}_{\mathrm{E}_{\mathrm{q}}(2)}$ is dense in $B_{\mathrm{E}_{\mathrm{q}}(2)}, \mathcal{C}:=j_{\mathrm{C}(\mathbb{T})}\left(\mathrm{C}(\mathbb{T}) j_{B_{\mathrm{E}_{\mathrm{q}}(2)}}\left(\mathcal{B}_{\mathrm{E}_{\mathrm{q}}(2)}\right)\right.$ is also dense in $C$ and the equation (6.15) coincides with the following:

$$
\Delta_{C}(c)=\lim _{k \rightarrow \infty}\left(\tilde{\tau}^{k} \otimes \tilde{\tau}^{k}\right) \Delta_{\mathrm{U}_{\mathrm{q}}(2)}\left(\tilde{\tau}^{-k}(c)\right) \quad \text { for } c \in \mathcal{C} .
$$

For every $k \in \mathbb{Z}$, the automorphisms $\tilde{\tau}^{k}$ act trivially on the first factor of $C=$ $\mathrm{C}(\mathbb{T}) \bigotimes_{\mathrm{R}} B_{\mathrm{E}_{\mathrm{q}}(2)}$. Subsequently, (6.16) becomes the equality of the restrictions of $\Delta_{C}$ and $\Delta_{\mathrm{U}_{\mathrm{q}}(2)}$ on the common closed quantum subgroup $\mathbb{T}$ for $c=j_{\mathrm{C}(\mathbb{T})}(z)$.

Restriction of the faithful representation $j_{B_{\mathrm{E}_{\mathrm{q}}(2)}} \in \operatorname{Mor}\left(B_{\mathrm{E}_{\mathrm{q}}(2)}, \mathbb{K}\left(\mathcal{H} \otimes \mathcal{L}_{\mathrm{E}_{\mathrm{q}}(2)}\right)\right)$ on $\mathrm{C}(\mathbb{T})$ is given by $j_{B_{\mathrm{E}_{(}(2)}}(v)=1_{\mathcal{H}} \otimes v$. Then $\tilde{\tau}^{k}(c)=\left(1_{\mathcal{H}} \otimes v^{k}\right) c\left(1_{\mathcal{H}} \otimes v^{-k}\right)$ for $c \in C \subset \mathbb{B}\left(\mathcal{H} \otimes \mathcal{L}_{\mathrm{E}_{\mathrm{q}}(2)}\right)$ and $k \in \mathbb{Z}$. It is also easy to verify that $v \otimes 1_{\mathcal{H}}$ commutes with $\mathbb{U}, 1_{\mathcal{H}} \otimes v$ commutes with $\hat{\mathbb{V}}$. These imply

$\left(\tilde{\tau}^{k} \otimes \tilde{\tau}^{k}\right) \Psi(x)=\Psi\left(\left(\operatorname{id}_{\mathrm{C}(\mathbb{T})} \bigotimes_{\mathrm{R}} \tau^{k} \otimes_{\mathrm{R}} \tau^{k}\right)(x)\right) \quad$ for all $x \in \mathrm{C}(\mathbb{T}) \bigotimes_{\mathrm{R}} B_{\mathrm{E}_{\mathrm{q}}(2)} \bigotimes_{\mathrm{R}} B_{\mathrm{E}_{\mathrm{q}}(2)}$.

Finally, using (6.8) we verify (6.16) for $c=j_{B_{\mathrm{E}_{\mathrm{q}}(2)}}(b)$ with $b \in \mathcal{B}_{\mathrm{E}_{\mathrm{q}}(2)}$

$$
\begin{aligned}
& \Delta_{C}\left(j_{B_{\mathrm{E}_{\mathrm{q}}(2)}}(b)\right)=\Psi\left(1_{\mathrm{C}(\mathbb{T})} \otimes_{\mathrm{R}} \Delta_{\mathrm{E}_{\mathrm{q}}(2)}(b)\right) \\
& =\Psi\left(1_{\mathrm{C}(\mathbb{T})} \bigotimes_{\mathrm{R}} \lim _{k \rightarrow \infty}\left(\left(\tau^{k} \bigotimes_{\mathrm{R}} \tau^{k}\right) \Delta_{\mathrm{SU}_{\mathrm{q}}(2)}\left(\tau^{-k}(b)\right)\right)\right) \\
& =\lim _{k \rightarrow \infty} \Psi\left(1_{\mathrm{C}(\mathbb{T})} \otimes_{\mathrm{R}}\left(\left(\tau^{k} \otimes_{\mathrm{R}} \tau^{k}\right) \Delta_{\mathrm{SU}_{\mathrm{q}}(2)}\left(\tau^{-k}(b)\right)\right)\right) \\
& =\lim _{k \rightarrow \infty}\left(\left(\tilde{\tau}^{k} \otimes \tilde{\tau}^{k}\right) \Psi\left(1_{\mathrm{C}(\mathbb{T})} \otimes_{\mathrm{R}} \Delta_{\mathrm{SU}_{\mathrm{q}}(2)}\left(\tau^{-k}(b)\right)\right)\right) \\
& =\lim _{k \rightarrow \infty}\left(\left(\tilde{\tau}^{k} \otimes \tilde{\tau}^{k}\right) \Delta_{\mathrm{U}_{\mathrm{q}}(2)}\left(j_{B_{\mathrm{SU}_{\mathrm{q}}(2)}}\left(\tau^{-k}(b)\right)\right)\right) \\
& =\lim _{k \rightarrow \infty}\left(\left(\tilde{\tau}^{k} \otimes \tilde{\tau}^{k}\right) \Delta_{\mathrm{U}_{\mathrm{q}}(2)}\left(\tilde{\tau}^{-k}\left(j_{B_{\mathrm{SU}_{\mathrm{q}}(2)}}(b)\right)\right)\right) .
\end{aligned}
$$

\section{Appendix A. Yetter-Drinfeld Representation CATEgory over} QUASITRIANGULAR QUANTUM GROUPS

Let $\mathbb{G}=\left(A, \Delta_{A}\right)$ be a quantum group, $\widehat{\mathbb{G}}=\left(\hat{A}, \hat{\Delta}_{A}\right)$ be its dual, and $\mathrm{W} \in$ $\mathcal{U}(\hat{A} \otimes A)$ be the reduced bicharacter.

A $\mathbb{G}$-Yetter-Drinfeld representation is a triple $(\mathcal{L}, \mathrm{U}, \mathrm{V})$ consisting of a Hilbert space $\mathcal{L}$, representations $\mathrm{U}$ and $\mathrm{V}$ of $\mathbb{G}$ and $\widehat{\mathbb{G}}$ on $\mathcal{L}$ subject to the commutation relation:

$$
\mathrm{V}_{12} \mathrm{U}_{13} \mathrm{~W}_{23}=\mathrm{W}_{23} \mathrm{U}_{13} \mathrm{~V}_{12} \quad \text { in } \mathcal{U}(\mathbb{K}(\mathcal{L}) \otimes \hat{A} \otimes A) .
$$


A morphism between $\mathbb{G}$-Yetter-Drinfeld representations $\left(\mathcal{L}_{1}, \mathrm{U}^{1}, \mathrm{~V}^{1}\right)$ and $\left(\mathcal{L}_{2}, \mathrm{U}^{2}, \mathrm{~V}^{2}\right)$ is an element $t \in \mathbb{B}\left(\mathcal{L}_{1}, \mathcal{L}_{2}\right)$ such that $t \in \operatorname{Hom}^{\mathbb{G}}\left(\mathrm{U}^{1}, \mathrm{U}^{2}\right)$ in $\mathfrak{R e p}(\mathbb{G})$ and $t \in$ $\operatorname{Hom}^{\widehat{\mathbb{G}}}\left(\mathrm{V}^{1}, \mathrm{~V}^{2}\right)$ in $\mathfrak{R e p}(\widehat{\mathbb{G}})$, respectively. Let $\mathcal{Y} \mathcal{D} \mathfrak{R e p}(\mathbb{G})$ denotes the category of $\mathbb{G}$-Yetter-Drinfeld representations. It is easy to verify that $\oplus$ operation on $\mathcal{Y} \mathcal{D} \mathfrak{R e p}(\mathbb{G})$ defined by $\left(\mathcal{L}_{1}, \mathrm{U}^{1}, \mathrm{~V}^{1}\right) \oplus\left(\mathcal{L}_{2}, \mathrm{U}^{2}, \mathrm{~V}^{2}\right):=\left(\mathcal{L}_{1} \otimes \mathcal{L}_{2}, \mathrm{U}^{1} \oplus \mathrm{U}^{2}, \mathrm{~V}^{1} \oplus \mathrm{V}^{2}\right)$ is a tensor product with tensor unit $\mathbb{C}$. This category is already a unitarily braided monoidal category, see [14, Proposition 3.2].

Proposition A.2. Let $\mathbb{G}=\left(A, \Delta_{A}\right)$ be a quasitriangular quantum group with an $R$-matrix $\mathrm{R} \in \mathcal{U}(\hat{A} \otimes \hat{A})$. For any object $(\mathcal{L}, \mathrm{U})$ in $\mathfrak{R e p}(\mathbb{G})$ the $R$-matrix $\mathrm{R}$ induces a canonical object $(\mathcal{L}, \mathrm{V})$ in $\mathfrak{R e p}(\widehat{\mathbb{G}})$ such that $(\mathcal{L}, \mathrm{U}, \mathrm{V})$ becomes an object in $\mathcal{Y} \mathcal{D} \mathfrak{R e p}(\mathbb{G})$. Moreover, the construction gives an injective braided monoidal functor $\mathcal{F}: \mathfrak{R e p}(\mathbb{G}) \rightarrow \mathcal{Y D} \mathfrak{R e p}(\mathbb{G})$ that maps $(\mathcal{L}, \mathrm{U})$ to $(\mathcal{L}, \mathrm{U}, \mathrm{V})$ and leaves the morphisms unchanged.

Proof. Since $\mathrm{R} \in \mathcal{U}(\hat{A} \otimes \hat{A})$ is a bicharacter, it is also a quantum group homomorphism from $\mathbb{G}$ to $\widehat{\mathbb{G}}$. Let $\Delta_{R} \in \operatorname{Mor}(A, A \otimes \hat{A})$ be the right quantum group homomorphism associated to it [12, Theorem 5.3]. Let $(\mathcal{L}, \mathrm{U})$ be an object in $\mathfrak{R e p}(\mathbb{G})$. Consider the unitary $\widetilde{V}:=\mathrm{U}_{12}^{*}\left(\left(\operatorname{id}_{\mathbb{K}(\mathcal{L})} \otimes \Delta_{R}\right) \mathrm{U}\right) \in \mathcal{U}(\mathbb{K}(\mathcal{L}) \otimes A \otimes \hat{A})$. In particular, $\Delta_{R}$ satisfies $\left(\Delta_{A} \otimes \operatorname{id}_{\hat{A}}\right) \circ \Delta_{R}=\left(\operatorname{id}_{A} \otimes \Delta_{R}\right) \circ \Delta_{A}$. Using the last equation and the fact the $\mathrm{U}$ is a representation, we compute

$$
\begin{aligned}
\left(\operatorname{id}_{\mathbb{K}(\mathcal{L})} \otimes \Delta_{A} \otimes \operatorname{id}_{\hat{A}}\right) \widetilde{V} & =\left(\left(\operatorname{id}_{\mathbb{K}(\mathcal{L})} \otimes \Delta_{A}\right) \mathrm{U}^{*}\right)_{123}\left(\left(\operatorname{id}_{\mathbb{K}(\mathcal{L})} \otimes\left(\left(\Delta_{A} \otimes \operatorname{id}_{\hat{A}}\right) \Delta_{R}\right)\right) \mathrm{U}\right) \\
& =\mathrm{U}_{13}^{*} \mathrm{U}_{12}^{*}\left(\left(\operatorname{id}_{\mathbb{K}(\mathcal{L})} \otimes\left(\left(\operatorname{id}_{A} \otimes \Delta_{R}\right) \Delta_{A}\right)\right) \mathrm{U}\right) \\
& =\mathrm{U}_{13}^{*} \mathrm{U}_{12}^{*}\left(\left(\operatorname{id}_{\mathbb{K}(\mathcal{L})} \otimes \operatorname{id}_{A} \otimes \Delta_{R}\right) \mathrm{U}_{12} \mathrm{U}_{13}\right) \\
& =\mathrm{U}_{13}^{*}\left(\left(\operatorname{id}_{\mathbb{K}(\mathcal{L})} \otimes \Delta_{R}\right) \mathrm{U}\right)_{134}=\widetilde{V}_{134} .
\end{aligned}
$$

By [12, Corollary 2.2] the second leg of $\widetilde{V}$ is trivial; hence there is a unique element $\mathrm{V} \in \mathcal{U}(\mathbb{K}(\mathcal{L}) \otimes \hat{A})$ such that

$$
\left(\operatorname{id}_{\mathbb{K}(\mathcal{L})} \otimes \Delta_{R}\right) \mathrm{U}=\mathrm{U}_{12} \mathrm{~V}_{13} \quad \text { in } \mathcal{U}(\mathbb{K}(\mathcal{L}) \otimes A \otimes \hat{A}) .
$$

Also, the first part of the proof of [12, Theorem 5.3] shows that $(\mathcal{L}, \mathrm{V})$ is an object in $\mathfrak{R e p}(\widehat{\mathbb{G}})$.

Now we show that $(\mathcal{L}, \mathrm{U}, \mathrm{V})$ is an object in $\mathcal{Y} \mathcal{D} \mathfrak{R e p}(\mathbb{G})$. The second condition in (2.4) and the $R$-matrix condition (2.10) together imply

$$
\begin{aligned}
\mathrm{W}_{23} \mathrm{~W}_{13} \mathrm{R}_{12}=\left(\left(\hat{\Delta}_{A} \otimes \mathrm{id}_{A}\right) \mathrm{W}\right) \mathrm{R}_{12}=\mathrm{R}_{12}\left(\left(\sigma \circ \hat{\Delta}_{A} \otimes \mathrm{id}_{A}\right) \mathrm{W}\right) & =\mathrm{R}_{12}\left(\sigma_{12}\left(\mathrm{~W}_{23} \mathrm{~W}_{13}\right)\right) \\
& =\mathrm{R}_{12} \mathrm{~W}_{13} \mathrm{~W}_{23} .
\end{aligned}
$$

The bijective correspondence between $\mathrm{R}$ and $\Delta_{R}$ in [12, Equation 32], the first condition in (2.4) and the last identity give

$$
\begin{aligned}
\left.\mathrm{W}_{34}\left(\sigma_{34}\left(\mathrm{id}_{\hat{A}} \otimes\left(\Delta_{A} \otimes \mathrm{id}_{A}\right) \Delta_{R}\right) \mathrm{W}\right)\right) \mathrm{W}_{34}^{*} & =\mathrm{W}_{34}\left(\sigma_{34}\left(\mathrm{~W}_{12} \mathrm{~W}_{13} \mathrm{R}_{14}\right)\right) \mathrm{W}_{34}^{*} \\
& =\mathrm{W}_{34} \mathrm{~W}_{12} \mathrm{~W}_{14} \mathrm{R}_{13} \mathrm{~W}_{34}^{*} \\
& =\mathrm{W}_{12} \mathrm{~W}_{34} \mathrm{~W}_{14} \mathrm{R}_{13} \mathrm{~W}_{34}^{*} \\
& =\mathrm{W}_{12} \mathrm{R}_{13} \mathrm{~W}_{14} \\
& =\left(\mathrm{id}_{\hat{A}} \otimes\left(\Delta_{R} \otimes \mathrm{id}_{A}\right) \Delta_{A}\right) \mathrm{W} .
\end{aligned}
$$

Then slicing the first leg of the last computation by $\omega \in \hat{A}^{\prime}$ we obtain

$$
\mathrm{W}_{23}\left(\sigma_{23}\left(\left(\Delta_{A} \otimes \operatorname{id}_{A}\right) \Delta_{R}(a)\right)\right) \mathrm{W}_{23}^{*}=\left(\Delta_{R} \otimes \operatorname{id}_{A}\right) \Delta_{A}(a) \text { for all } a \in A \text {. }
$$


Using this we now compute

$$
\begin{aligned}
\mathrm{U}_{12} \mathrm{~V}_{13} \mathrm{U}_{14} \mathrm{~W}_{34} & =\left(\left(\mathrm{id}_{\mathbb{K}(\mathcal{L})} \otimes\left(\Delta_{R} \otimes \mathrm{id}_{A}\right) \Delta_{A}\right) \mathrm{U}\right) \mathrm{W}_{34} \\
& =\mathrm{W}_{34}\left(\sigma_{34}\left(\left(\mathrm{id}_{\mathbb{K}(\mathcal{L})} \otimes\left(\Delta_{A} \otimes \mathrm{id}_{A}\right) \Delta_{R}\right) \mathrm{U}\right)\right) \\
& =\mathrm{W}_{34}\left(\sigma_{34}\left(\mathrm{U}_{12} \mathrm{U}_{13} \mathrm{~V}_{14}\right)\right)=\mathrm{W}_{34} \mathrm{U}_{12} \mathrm{U}_{14} \mathrm{~V}_{13}=\mathrm{U}_{12} \mathrm{~W}_{34} \mathrm{U}_{14} \mathrm{~V}_{13} .
\end{aligned}
$$

Cancelling the unitary $U_{12}$ on both sides of the last equation we obtain (A.1); hence $(\mathcal{L}, \mathrm{U}, \mathrm{V})$ is an object in $\mathcal{Y} \mathcal{D} \mathfrak{R e p}(\mathbb{G})$.

Suppose $\left(\mathcal{L}_{i}, \mathrm{U}^{i}\right)$ and $\left(\mathcal{L}_{i}, \mathrm{~V}^{i}\right)$ are objects in $\mathfrak{R e p}(\mathbb{G})$ and $\mathfrak{R e p}(\widehat{\mathbb{G}})$ respectively, induced by the $R$-matrix of $\mathbb{G}$ for $i=1,2$. Let $t \in \operatorname{Hom}^{\mathbb{G}}\left(\mathrm{U}^{1}, \mathrm{U}^{2}\right)$ in $\mathfrak{R e p}(\mathbb{G})$. Then

$$
\begin{aligned}
\mathrm{U}_{12}^{2}\left(t \otimes 1_{A \otimes \hat{A}}\right) \mathrm{V}_{13}^{1}=\left(t \otimes 1_{A \otimes \hat{A}}\right) \mathrm{U}_{12}^{1} \mathrm{~V}_{13}^{1} & =\left(t \otimes \Delta_{R}\right) \mathrm{U} \\
& =\left(\left(\operatorname{id}_{\mathbb{K}\left(\mathcal{L}_{2}\right)} \otimes \Delta_{R}\right) \mathrm{U}^{2}\right)\left(t \otimes 1_{A \otimes \hat{A}}\right) \\
& =\mathrm{U}_{12}^{2} \mathrm{~V}_{13}^{2}\left(t \otimes 1_{A \otimes \hat{A}}\right) .
\end{aligned}
$$

Cancelling the unitary $\mathrm{U}_{12}^{2}$ on both sides of the last equation we get $t \in \operatorname{Hom}^{\widehat{\mathbb{G}}}\left(\mathrm{V}^{1}, \mathrm{~V}^{2}\right)$ in $\mathfrak{R e p}(\widehat{\mathbb{G}})$. Thus we have an injective functor $\mathcal{F}: \mathfrak{R e p}(\mathbb{G}) \rightarrow \mathcal{Y} \mathcal{D} \mathfrak{R e p}(\mathbb{G})$ that maps objects $(\mathcal{L}, \mathrm{U}) \rightarrow(\mathcal{L}, \mathrm{U}, \mathrm{V})$ and leaves the morphisms unchanged. Furthermore, $\mathcal{F}$ preserves the tensor product of representations:

$$
\begin{aligned}
\left(\mathrm{id}_{\mathbb{K}\left(\mathcal{L}_{1} \otimes \mathcal{L}_{2}\right)} \otimes \Delta_{R}\right) \mathrm{U}^{1} \oplus \mathrm{U}^{2} & =\left(\mathrm{id}_{\mathbb{K}\left(\mathcal{L}_{1} \otimes \mathcal{L}_{2}\right)} \otimes \Delta_{R}\right)\left(\mathrm{U}_{13}^{1} \mathrm{U}_{23}^{2}\right) \\
& =\mathrm{U}_{13}^{1} \mathrm{~V}_{14}^{1} \mathrm{U}_{23}^{2} \mathrm{~V}_{24}^{2} \\
& =\mathrm{U}_{13}^{1} \mathrm{U}_{23}^{2} \mathrm{~V}_{14}^{1} \mathrm{~V}_{24}^{2}=\left(\mathrm{U}^{1} \oplus \mathrm{U}^{2}\right)_{123}\left(\mathrm{~V}^{1} \oplus \mathrm{V}^{2}\right)_{124}
\end{aligned}
$$

In particular, the proof of [14, Theorem 5.3] shows that $\mathcal{F}$ preserves the braidings. Therefore, $\mathcal{F}$ is an injective braided monoidal structure preserving functor.

\section{REFERENCES}

[1] Saad Baaj, Représentation régulière du groupe quantique $E_{\mu}(2)$ de Woronowicz, C. R. Acad. Sci. Paris Sér. I Math. 314 (1992), no. 13, 1021-1026, available at http://gallica.bnf.fr/ark:/12148/bpt6k58688425/f1025.item. MR1168528

[2] Saad Baaj and Georges Skandalis, Unitaires multiplicatifs et dualité pour les produits croisés de $C^{*}$-algèbres, Ann. Sci. École Norm. Sup. (4) 26 (1993), no. 4, 425-488, available at http://www.numdam.org/item?id=ASENS_1993_4_26_4_425_0 MR1235438

[3] Enrico Celeghini, Riccardo Giachetti, Emanuele Sorace, and Marco Tarlini, Threedimensional quantum groups from contractions of $\mathrm{SU}(2)_{q}$, J. Math. Phys. 31 (1990), no. 11, 2548-2551, doi: 10.1063/1.529000 MR1075731

[4] - The quantum Heisenberg group $H(1)_{q}$, J. Math. Phys. 32 (1991), no. 5, 1155-1158, doi: 10.1063/1.529311 MR1103466

[5] - The three-dimensional Euclidean quantum group $E(3)_{q}$ and its $R$-matrix, J. Math. Phys. 32 (1991), no. 5, 1159-1165, doi: 10.1063/1.529312 MR1103467

[6] Matthew Daws, Paweł Kasprzak, Adam Skalski, and Piotr M. Sołtan, Closed quantum subgroups of locally compact quantum groups, Adv. Math. 231 (2012), no. 6, 3473-3501, doi: 10.1016/j.aim.2012.09.002 MR2980506

[7] Erdal Inönü and Eugene P. Wigner, On the contraction of groups and their representations, Proc. Nat. Acad. Sci. U.S.A. 39 (1953), 510-524, doi: 10.1073/pnas.39.6.510 MR55352

[8] Paweł Kasprzak, Ralf Meyer, Sutanu Roy, and Stanisław L. Woronowicz, Braided quantum $\mathrm{SU}(2)$ groups, J. Noncommut. Geom. 10 (2016), no. 4, 1611-1625, doi: 10.4171/JNCG/268 MR3597153

[9] Shahn Majid, Cross products by braided groups and bosonization, J. Algebra 163 (1994), no. 1, 165-190, doi: 10.1006/jabr.1994.1011 MR1257312

[10] S. Majid, Double-bosonization of braided groups and the construction of $U_{q}(\mathfrak{g})$, Math. Proc. Cambridge Philos. Soc. 125 (1999), no. 1, 151-192, doi: 10.1017/S0305004198002576 MR1645545

[11] Shahn Majid, Braided-Lie bialgebras, Pacific J. Math. 192 (2000), no. 2, 329-356, doi: 10.2140/pjm.2000.192.329 MR1744574 
[12] Ralf Meyer, Sutanu Roy, and Stanisław L. Woronowicz, Homomorphisms of quantum groups, Münster J. Math. 5 (2012), 1-24, available at http://nbn-resolving.de/urn:nbn:de:hbz:6-88399662599. MR3047623

[13] Quantum group-twisted tensor products of $C^{*}$-algebras, Internat. J. Math. 25 (2014), no. 2, 1450019, 37, doi: 10.1142/S0129167X14500190 MR3189775

[14] _ Quantum group-twisted tensor products of $\mathrm{C}^{*}$-algebras. II, J. Noncommut. Geom. 10 (2016), no. 3, 859-888, doi: 10.4171/JNCG/250 MR3554838

[15] Ralf Meyer, Sutanu Roy, and Stanisław Lech Woronowicz, Semidirect products of C* ${ }^{*}$-quantum groups: multiplicative unitaries approach, Comm. Math. Phys. 351 (2017), no. 1, 249-282, doi: 10.1007/s00220-016-2727-3 MR3613504

[16] Ryszard Nest and Christian Voigt, Equivariant Poincaré duality for quantum group actions, J. Funct. Anal. 258 (2010), no. 5, 1466-1503, doi: 10.1016/j.jfa.2009.10.015 MR2566309

[17] David E. Radford, The structure of Hopf algebras with a projection, J. Algebra 92 (1985), no. 2, 322-347, doi: 10.1016/0021-8693(85)90124-3 MR778452

[18] Sutanu Roy, $\mathrm{C}^{*}$-quantum groups with projection, Ph.D. Thesis, Georg-August Universität Göttingen, 2013, http://hdl.handle.net/11858/00-1735-0000-0022-5EF9-0

[19] (2016), available at https://arxiv.org/abs/1601.00169v4

[20] Piotr M. Sołtan, Podleś spheres for the braided quantum SU(2), Linear Algebra Appl. 591 (2020), 169-204, doi: 10.1016/j.laa.2020.01.011 MR4053085

[21] Piotr M. Soltan and Stanisław L. Woronowicz, From multiplicative unitaries to quantum groups. II, J. Funct. Anal. 252 (2007), no. 1, 42-67, doi: 10.1016/j.jfa.2007.07.006 MR2357350

[22] Stanisław L. Woronowicz, Quantum E(2) group and its Pontryagin dual, Lett. Math. Phys. 23 (1991), no. 4, 251-263, doi: 10.1007/BF00398822 MR1152695

[23] - Unbounded elements affiliated with $C^{*}$-algebras and noncompact quantum groups, Comm. Math. Phys. 136 (1991), no. 2, 399-432. MR1096123

[24] - Operator equalities related to the quantum E(2) group, Comm. Math. Phys. 144 (1992), no. 2, 417-428. MR1152380

[25] , Quantum $\mathrm{SU}(2)$ and $E(2)$ groups. Contraction procedure, Comm. Math. Phys. 149 (1992), no. 3, 637-652. MR1186047

[26] $\longrightarrow C^{*}$-algebras generated by unbounded elements, Rev. Math. Phys. 7 (1995), no. 3, 481-521, doi: 10.1142/S0129055X95000207 MR1326143

[27] _ From multiplicative unitaries to quantum groups, Internat. J. Math. 7 (1996), no. 1, 127-149, doi: 10.1142/S0129167X96000086 MR1369908

[28] — Quantum "az+b" group on complex plane, Internat. J. Math. 12 (2001), no. 4, 461-503, doi: 10.1142/S0129167X01000836 MR1841400

[29] Stanisław L. Woronowicz and S. Zakrzewski, Quantum ' $a x+b$ ' group, Rev. Math. Phys. 14 (2002), no. 7-8, 797-828, doi: 10.1142/S0129055X02001405 MR1932667

Email address: atibur.rahaman@niser.ac.in

School of Mathematical Sciences, National Institute of Science Education and ReSEARCh Bhubaneswar, HBNI, Jatni, 752050, India

Email address: sutanu@niser.ac.in

School of Mathematical Sciences, National Institute of Science Education and ReSEARCh BHubaneswar, HBNi, Jatni, 752050, India 